\title{
Modeling and Simulation of Variable Mass, Flexible Structures
}

\section{Patrick Tobbe, Alex Matras, Heath Wilson - Dynamic Concepts, Inc.}

The advent of the new Ares I launch vehicle has highlighted the need for advanced dynamic analysis tools for variable mass, flexible structures. This system is composed of interconnected flexible stages or components undergoing rapid mass depletion through the consumption of solid or liquid propellant. In addition to large rigid body configuration changes, the system simultaneously experiences elastic deformations. In most applications, the elastic deformations are compatible with linear straindisplacement relationships and are typically modeled using the assumed modes technique. The deformation of the system is approximated through the linear combination of the products of spatial shape functions and generalized time coordinates. Spatial shape functions are traditionally composed of normal mode shapes of the system or even constraint modes and static deformations derived from finite element models of the system. Equations of motion for systems undergoing coupled large rigid body motion and elastic deformation have previously been derived through a number of techniques [1]. However, in these derivations, the mode shapes or spatial shape functions of the system components were considered constant. But with the Ares I vehicle, the structural characteristics of the system are changing with the mass of the system.

Previous approaches to solving this problem involve periodic updates to the spatial shape functions or interpolation between shape functions based on system mass or elapsed mission time. These solutions often introduce misleading or even unstable numerical transients into the system. Plus, interpolation on a shape function is not intuitive. This paper presents an approach in which the shape functions are held constant and operate on the changing mass and stiffness matrices of the vehicle components. Each vehicle stage or component finite element model is broken into dry structure and propellant models. A library of propellant models is used to describe the distribution of mass in the fuel tank or Solid Rocket Booster (SRB) case for various propellant levels. Based on the mass consumed by the liquid engine or SRB, the appropriate propellant model is coupled with the dry structure model for the stage. Then using vehicle configuration data, the integrated vehicle model is assembled and operated on by the constant system shape functions. The system mode shapes and frequencies can then be computed from the resulting generalized mass and stiffness matrices for that mass configuration. The rigid body mass properties of the vehicle are derived from the integrated vehicle model. The coupling terms between the vehicle rigid body motion and elastic deformation are also updated from the constant system shape functions and the integrated vehicle model. This approach was first used to analyze variable mass spinning beams and then prototyped into a generic dynamics simulation engine. The resulting code was tested against Crew Launch Vehicle (CLV-)class problems worked in the TREETOPS simulation package and by Wilson [2].

The Ares I System Integration Laboratory (SIL) is currently being developed at the Marshall Space Flight Center (MSFC) to test vehicle avionics hardware and software in a hardware-in-the-loop (HWIL) environment and certify that the integrated system is

prepared for flight. The Ares I SIL utilizes the Ares Real-Time Environment for Modeling, Integration, and Simulation (ARTEMIS) tool to simulate the launch vehicle 
and stimulate avionics hardware. Due to the presence of vehicle control system filters and the thrust oscillation suppression system, which are tuned to the structural characteristics of the vehicle, ARTEMIS must incorporate accurate structural models of the Ares I launch vehicle. The ARTEMIS core dynamics simulation models the highly coupled nature of the vehicle flexible body dynamics, propellant slosh, and vehicle nozzle inertia effects combined with mass and flexible body properties that vary significantly with time during the flight. All forces that act on the vehicle during flight must be simulated, including deflected engine thrust force, spatially distributed aerodynamic forces, gravity, and reaction control jet thrust forces. These forces are used to excite an integrated flexible vehicle, slosh, and nozzle dynamics model for the vehicle stack that simulates large rigid body translations and rotations along with small elastic deformations. Highly effective matrix math operations on a distributed, threaded high-performance simulation node allow ARTEMIS to retain up to 30 modes of flex for real-time simulation. Stage elements that separate from the stack during flight are propagated as independent rigid six degrees of freedom (6DOF) bodies. This paper will present the formulation of the resulting equations of motion, solutions to example problems, and describe the resulting dynamics simulation engine within ARTEMIS. 


\title{
Modeling and Simulation of Variable Mass, Flexible Structures
}

\author{
Patrick A. Tobbe, Ph. D. ${ }^{1}$, Alex L. Matras, Ph. D. ${ }^{2}$, and Heath E. Wilson ${ }^{2}$ \\ Dynamic Concepts, Inc., Huntsville, AL, 35806
}

\begin{abstract}
The advent of the new Ares I launch vehicle has highlighted the need for advanced dynamics analysis tools for variable mass, flexible structures. Traditional flexible body simulation tools are based on the assumed modes technique in which component modes are constant. However, for variable mass systems, component and system modes vary with mass. This paper presents a technique that accurately simulates the response of a variable mass, flexible body while utilizing a constant set of shape functions. The approach allows for continued use of current flexible body simulation dynamics engines. The Ares Real-Time Environment for Modeling, Integration, and Simulation (ARTEMIS) has been developed for use by the Ares I launch vehicle System Integration Laboratory at the Marshall Space Flight Center. ARTEMIS utilizes the proposed approach in a real-time environment. Variable mass, flexible body test cases will be solved and presented from ARTEMIS and the multibody simulation tool TREETOPS.
\end{abstract}

\section{Nomenclature}

$f_{0}^{\alpha} \quad=$ force and moment vector for component $\alpha$

$K_{0} \quad=$ block diagonal of all component stiffness matrices

$k_{0}{ }^{\alpha} \quad=$ stiffness matrix

$M_{0} \quad=$ block diagonal of all component mass matrices

$m_{0}{ }^{\alpha} \quad=$ mass matrix for component $\alpha$

$\dot{m}_{p}^{i} \quad=$ propellant mass flow rate for $\mathrm{i}^{\text {th }}$ engine/thruster on component

$m_{c}{ }^{t} \quad=$ propellant mass consumed for $\mathrm{t}^{\text {th }}$ tank on component

$M_{d} \quad=$ dry component structural mass matrix

$M_{p} \quad=$ component propellant structural mass matrix

$M \quad=$ component mass matrix

$q \quad=\quad$ state vector of constrained system

$S \quad=$ constraint matrix

$T_{10}=$ transformation to sort dependent and independent degrees of freedom order

$u_{0}{ }^{\alpha}=$ state vector in arbitrary order for component $\alpha$

$u_{0} \quad=$ state vector in arbitrary order

$u_{1} \quad=$ state vector sorted into dependent and independent degrees of freedom

$V \quad=$ product of transformation, constraint and mode shape matrices

$v_{\alpha}=$ submatrix of $V$, partitioned to size of component $\alpha$

$\Gamma_{0} \quad=$ first mass integral

$\Gamma_{1} \quad=$ second mass integral

$\kappa=$ generalized stiffness matrix

$\mu \quad=$ generalized mass matrix

$\Phi \quad=$ mode shape matrix

${ }^{1}$ Chief Engineer, 6700 Odyssey Drive, Suite 202, AIAA member

${ }^{2}$ Engineer/Scientist, Simulation, Model, and Test Division, 6700 Odyssey Drive, Suite 202, AIAA member 1 American Institute of Aeronautics and Astronautics 


\section{Introduction}

$\mathrm{I}_{\mathrm{s}}^{\mathrm{n}}$ nnovative dynamics analysis tools are essential for modeling and simulation of variable mass, flexible structures such as the new Ares I launch vehicle. This system is composed of interconnected flexible stages or components undergoing rapid mass depletion through the consumption of solid or liquid propellant. In addition to large rigid body configuration changes, the system simultaneously experiences elastic deformations. In most applications, the elastic deformations are compatible with linear strain-displacement relationships and are typically modeled using the assumed modes technique. The deformation of the system is approximated through the linear combination of the products of spatial shape functions and generalized time coordinates. Spatial shape functions are traditionally composed of normal mode shapes of the system or even constraint modes and static deformations derived from finite element models of the system. Equations of motion for systems undergoing coupled large rigid body motion and elastic deformation have previously been derived through a number of techniques ${ }^{1}$. In these derivations, the mode shapes or spatial shape functions of the system components were considered constant; however, the structural characteristics of the Ares I vehicle change with the mass of the system.

Previous approaches to solving this problem involve periodic updates to the spatial shape functions or interpolation between shape functions based on system mass or elapsed mission time. The effects of these techniques have been studied ${ }^{2}$. These solutions often introduce misleading or even unstable numerical transients into the system. Plus, interpolation on a shape function is not intuitive. This paper presents an approach in which the shape functions are held constant and operate on the changing mass and stiffness matrices of the vehicle components. Each vehicle stage or component finite element model is broken into dry structure and propellant models. A library of propellant models is used to describe the distribution of mass in the fuel tanks or Solid Rocket Booster (SRB) for various propellant levels. Based on the mass consumed by the liquid engine or SRB, the appropriate propellant model is coupled with the dry structure model for the stage. Then, using vehicle configuration data, the integrated vehicle model is assembled and operated on by the constant system shape functions. The rigid body mass properties of the vehicle are derived from the integrated vehicle model. The coupling terms between the vehicle rigid body motion and elastic deformation are also updated from the constant system shape functions and the integrated vehicle model. This approach was first used to analyze variable mass spinning beams and then prototyped into a generic dynamics simulation engine. The resulting code was tested against Crew Launch Vehicle (CLV) class problems worked in the TREETOPS 3 simulation package.

The Ares I System Integration Laboratory (SIL) is currently being developed at the Marshall Space Flight Center (MSFC) to test vehicle avionics hardware and software in a hardware-in-the-loop (HWIL) environment and certify that the integrated system is prepared for flight. The Ares I SIL utilizes the Ares Real-Time Environment for Modeling, Integration, and Simulation (ARTEMIS) tool to simulate the launch vehicle and avionics hardware. Due to the presence of vehicle control system filters and the thrust oscillation suppression system, which are tuned to the structural characteristics of the vehicle, ARTEMIS must incorporate accurate structural models of the Ares I launch vehicle. The ARTEMIS core dynamics simulation models the highly coupled nature of the vehicle flexible body dynamics, propellant slosh, and vehicle nozzle inertia effects combined with mass and flexible body properties that vary significantly with time during the flight. All forces that act on the vehicle during flight must be simulated, including deflected engine thrust force, spatially distributed aerodynamic forces, gravity, and reaction control jet thrust forces. These forces are used to excite an integrated flexible vehicle, slosh, and nozzle dynamics model for the vehicle stack that simulates large rigid body translations and rotations along with small elastic deformations. Cache optimized matrix math operations on a high-performance, multiprocessor, multi-node simulation computer allow ARTEMIS to achieve real-time while retaining up to 30 flexural modes. Stages that separate from the stack during flight are propagated as independent, rigid six degree of freedom (6DOF) bodies. This paper will present the formulation of the resulting equations of motion, solutions to example problems, and describe the resulting dynamics simulation engine within ARTEMIS.

\section{Equations of Motion}

The vehicle equations of motion, which constitute the dynamics engine of the ARTEMIS simulation, were derived using Boltzmann-Hamel equations ${ }^{1}$. Some of that derivation will be repeated here. Boltzmann-Hamel equations, often referred to as Lagrange's equations in quasi-coordinates, are a modified form of Lagrange's equations which operate in a moving reference frame. The elastic deformations of each body will be approximated using the assumed modes technique. This method is a popular technique for modeling structural flexibility in multibody simulation packages. In this technique, the elastic deformation of each body is approximated through a linear 
combination of the products of shape functions and generalized time coordinates. The shape functions are typically a subset of normal modes derived from a finite element model of the component.

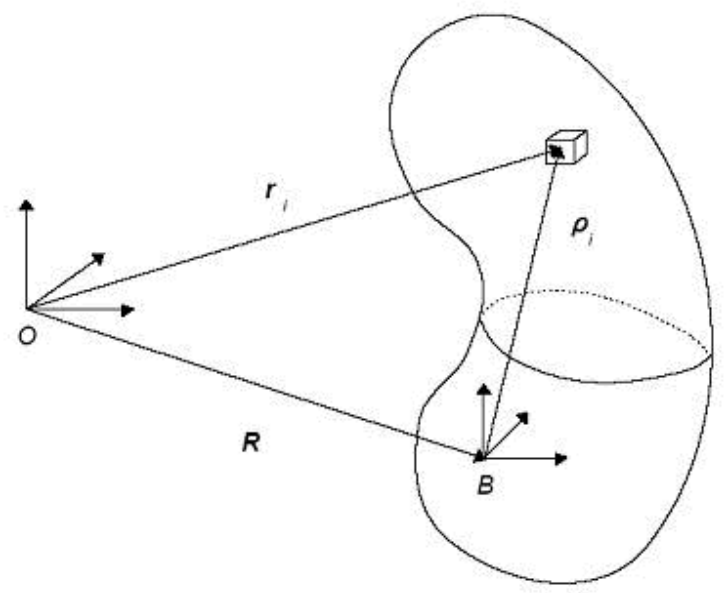

Figure 1. Undeformed Flexible Body

Shown in Fig. 1 is a generic flexible body with vectors describing the position of the $i^{\text {th }}$ nodal body relative to inertial space $O$ and a body fixed frame $B$. The nodal body has mass $m_{i}$ and an inertia tensor $I_{i}$. The location of the nodal body relative to the body fixed frame is denoted by $\rho_{i}$. In the deformed state of Fig. 2, the translational deformation of $i$ relative to $B$ is $\delta_{i}$ and the rotational deformation is $\theta_{i}$. These deformations are assumed to be small,

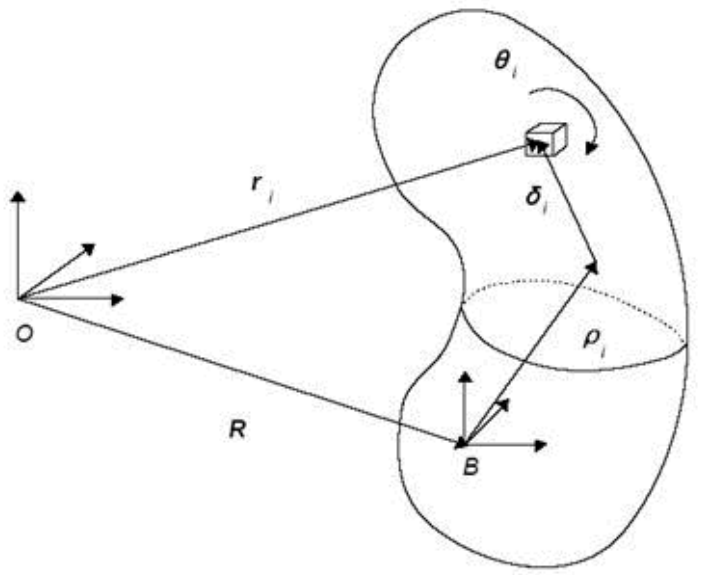

Figure 2. Deformed Flexible Body

as well as compatible with linear strain-displacement relations. The deformed nodal body position is

$$
r_{i}=R+\rho_{i}+\delta_{i}
$$

The inertial translational velocity of point $i$ is

$$
\dot{r}_{i}=\dot{R}+\omega \times\left(\rho_{i}+\delta_{i}\right)+\stackrel{\circ}{\delta_{i}}
$$


where $\omega$ is the angular velocity of the body frame $B$ and $\stackrel{\circ}{\delta}_{i}$ is the time rate of change of the deformation relative to $B$. The kinetic energy of the system can be written as

$$
\bar{T}=\sum_{i}\left\{\frac{1}{2} m_{i}\left(\dot{r}_{i} \cdot \dot{r}_{i}\right)+\frac{1}{2}\left(\omega+\dot{\theta}_{i}\right)^{T} I_{i}\left(\omega+\dot{\theta}_{i}\right)\right\}
$$

The system potential energy is the strain energy resulting from body deformations. It can be computed from a finite element stiffness matrix of the structure as

$$
V^{*}=\frac{1}{2}\left(\begin{array}{l}
\delta \\
\theta
\end{array}\right)^{T} K\left(\begin{array}{l}
\delta \\
\theta
\end{array}\right)-\lambda^{T} \alpha(q, t)
$$

where $K$ is the stiffness matrix, $\delta$ and $\theta$ are the nodal displacements, $\alpha(q, t)$ are the system constraint equations, and $\lambda$ is the array of Lagrange multipliers. The nodal displacements can be approximated using the assumed modes technique as

$$
\delta_{i}=\sum_{j} \phi_{i j} \eta_{j}
$$

and

$$
\theta_{i}=\sum_{j} \psi_{i j} \eta_{j}
$$

where $\phi_{i j}$ and $\psi_{i j}$ are the $j^{\text {th }}$ shape functions or modes at node $i$ and $\eta_{j}$ is the associated generalized coordinate. This is essentially a coordinate transformation from the finite element nodal displacements to a set of modal coordinates. The model is reduced in size by truncating the number of modes or shape functions used in the coordinate transformation. By inserting the energy expressions into Boltzmann-Hamel equations, the following equations of motion are derived.

$$
\begin{gathered}
{\left[\begin{array}{cccc}
M_{T} I_{3 x 3} & -\widetilde{\Gamma}_{0} \eta & \Gamma_{0} & -[B I] \alpha_{R_{0}^{T}}^{T} \\
\widetilde{\Gamma}_{0} \eta & I I & \Gamma_{1}+\Gamma_{2} & -\frac{1}{2} E \alpha_{\varepsilon_{B I}}^{T} \\
\Gamma_{0}^{T} & \left(\Gamma_{1}+\Gamma_{2}\right)^{T} & \mu & -\alpha_{\eta}^{T}
\end{array}\right]\left\{\begin{array}{c}
\circ \\
\dot{R}^{B} \\
\dot{\omega}^{B} \\
\ddot{\eta} \\
\lambda
\end{array}\right\}=} \\
\left\{\begin{array}{c}
-\omega^{B} \times\left\{M_{T} \dot{R}^{B}+2 \Gamma_{0} \dot{\eta}+\omega^{B} \times \Gamma_{0} \eta\right\}+F^{B} \\
-\left\{\Gamma_{0} \eta \times\left(\omega^{B} \times \dot{R}^{B}\right)\right\}-\omega^{B} \times I I \omega^{B}-\omega^{B} \times\left(\Gamma_{1} \dot{\eta}+\Gamma_{2 j} \dot{\eta}\right)+T^{B} \\
\phi_{i}^{T} F_{i}+\psi_{i}^{T} T_{i}+2 \dot{\Gamma}_{2 k}^{T} \omega^{B}+\Gamma_{0}^{T} \widetilde{\omega}^{B} \dot{R}^{B}-\mathrm{K} \eta+\frac{1}{2}\left\{\omega^{B} \cdot\left(2 I_{1 j}+I_{2 j k} \eta_{k}+I_{2 k j} \eta_{k}\right) \omega^{B}\right\}
\end{array}\right\}
\end{gathered}
$$

The off axis terms in the system mass matrix of Eq. (7) are defined as

$$
\begin{gathered}
\Gamma_{0 j}=\sum_{i=1}^{p} m_{i} \phi_{i j} \\
\Gamma_{1 j}=\sum_{i=1}^{p}\left(m_{i} \rho_{i} \times \phi_{i j}+I_{i} \psi_{i j}\right)
\end{gathered}
$$




$$
\begin{gathered}
I_{1 j}=\sum_{i=1}^{p} m_{i}\left(\rho_{i}^{T} \phi_{i j} 1-\phi_{i j} \rho_{i}^{T}\right) \\
\Gamma_{2 j k}=\sum_{i=1}^{p}\left(m_{i} \phi_{i j} \times \phi_{i k}\right) \\
I_{2 j k}=\sum_{i=1}^{p} m_{i}\left(\phi_{i j}^{T} \phi_{i k} 1-\phi_{i j} \phi_{i k}^{T}\right)
\end{gathered}
$$

These terms are often referred to as the mass integrals and are a function of the rigid body mass properties and selected shape functions. Generally, Eqs. (8) through (12) are evaluated using shape functions and a finite element mass matrix. The mass integral terms couple the rigid body motion to the structural deformation as characterized by the shape functions. If the shape functions are orthogonal to the rigid body modes of the system, the mass integral terms defined by Eqs. (8) and (9) are zero. Typically the higher order terms of Eqs. (10), (11), and (12) are ignored except for extremely flexible systems. The system natural frequencies and mode shapes can be computed from the system mass matrix (coefficient matrix of the acceleration vector in the equations of motion) and the generalized stiffness matrix (augmented with rows and columns of zeroes for the rigid body degrees of freedom).

\section{Variable Mass}

The changing structural characteristics of a variable mass system are difficult to model in a time domain simulation. In the previous derivation of equations of motion, the shape functions were considered constant. In order to account for variations in the structural characteristics, the equations of motion could be expanded to include timevarying terms for the shape functions. However, time derivatives of the shape functions are difficult to compute or measure and add significantly to the numerical computations. Alternatively, different sets of mode shapes for each mass distribution can be processed before running the simulation. These sets can be periodically updated or interpolated during the simulation using Eq. (7), which introduces discontinuities in the vehicle deformations. Finally, a constant set of shape vectors that describe the bending characteristics of the vehicle for the entire propellant burn can be built from combinations of the mode shapes derived from different mass distributions. In this approach, the stage mass and stiffness matrices are combined to build the integrated vehicle mass and stiffness matrices in physical coordinates. The vehicle mass properties, mass integrals, and generalized mass and stiffness matrices are computed from the integrated vehicle mass and stiffness matrices and constant shape functions each time step and updated in Eq. (7). Through these updates, the system equations of motion reflect the current structural characteristics of the vehicle in a continuous manner while using a constant set of shape vectors.

Vehicles simulated by the ARTEMIS dynamics engine are decomposed into stages. Each stage is further subdivided into a dry substructure and propellant or tank substructures. Finite element models of each of the stage substructures are created and the mass and stiffness matrices extracted. Multiple propellant or tank models are constructed for various levels of propellant. Within ARTEMIS, the propellant consumed by each substructure is tracked through integration of mass flow rates and the corresponding mass and stiffness matrices are updated using interpolation. The mass and stiffness matrices for the stages and integrated vehicle are assembled through substructure coupling techniques, based on the appropriate vehicle configuration, and are used to calculate mass properties, mass integrals, and generalized mass and stiffness matrices. System shapes are selected off line to characterize the integrated vehicle flexibility for the appropriate configuration of stages. These shapes are orthogonalized with respect to the vehicle geometric rigid body modes to avoid singularities caused through dependencies with explicit rigid body degrees of freedom defined in Eq. (7). Mass properties are found through geometric rigid body modes operating on the system mass matrix. The process to update the vehicle properties based on propellant consumption in ARTEMIS is shown in Fig. 3 for parallel processing. 


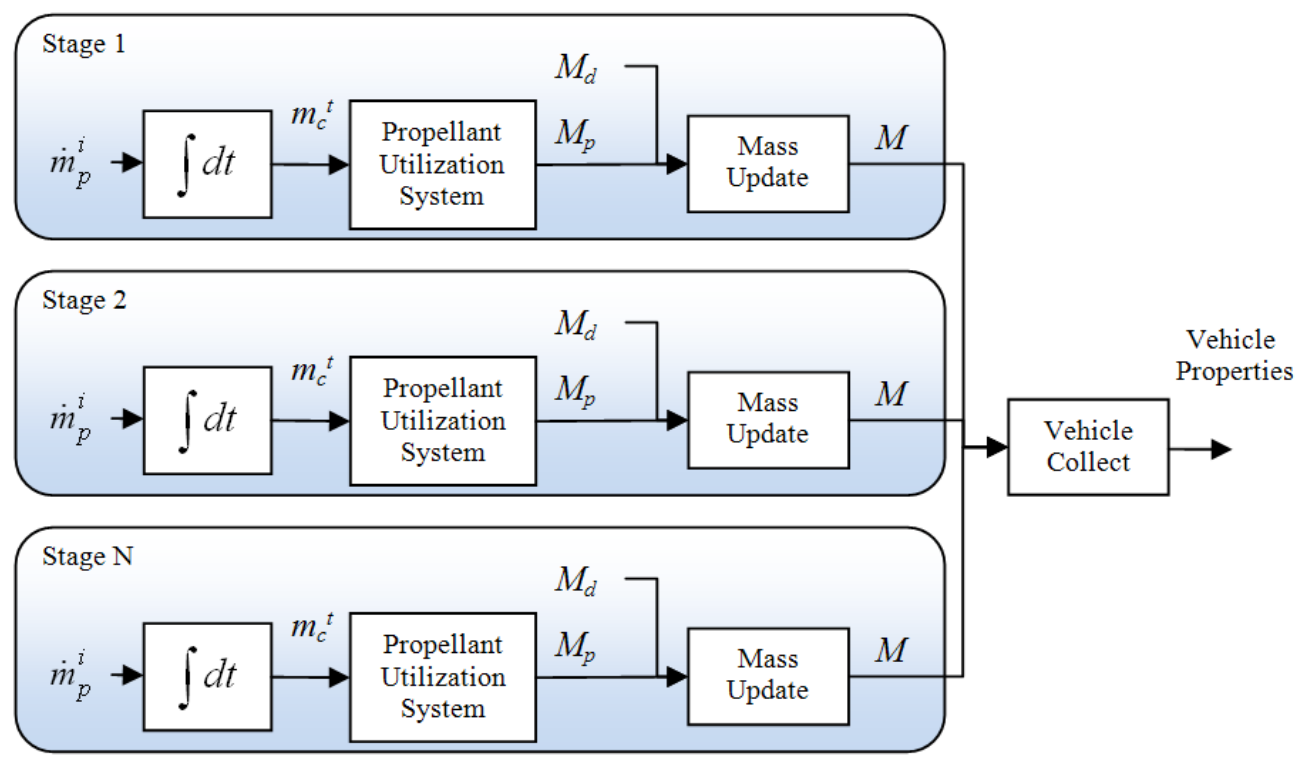

Figure 3. Vehicle Properties Calculation

\section{Algorithm Description}

The primary goals of the implementation of these methods are to derive equations in forms that yield a minimal number of run-time calculations, to parallelize tasks for efficient multithreading, and to design structures and algorithms to utilize the CPU cache as efficiently as possible.

The main technique used to reduce the number of run-time calculations is to partition the equations into terms that only need to be recomputed for a stage consuming mass, which is usually only one stage at a time. Additionally, the equations are written as a function of the individual stage mass and stiffness matrices before any transformations are applied. Thus, the equations reduce to simple matrix multiplications where most calculations may be performed one time at initialization and used for the duration of the simulation. The substructure coupling technique is particularly useful for deriving in this form.

\section{A. Substructure Coupling}

Substructure coupling is a convenient technique to assemble composite mass and stiffness matrices for structures from individual components. This method, also known as Component Mode Synthesis, is described in detail by Craig ${ }^{4}$. The formulation presented here has been modified to account for multiple components and to provide for more efficient calculations.

Consider a set of components (stages or substructures) $(\alpha, \beta, \gamma, \delta)$ where each component has a mass and stiffness matrix modeled by the following equation:

$$
m_{0}^{\alpha} \ddot{u}_{0}^{\alpha}+k_{0}^{\alpha} u_{0}^{\alpha}=f_{0}^{\alpha}
$$

The subscript 0 represents an arbitrary DOF order. In this notation, the superscript represents the component ( $\alpha$ in this case). The state vector is represented by $u$, the mass matrix by $m$, the stiffness matrix by $k$, and the external force and moments by $f$.

In order to perform substructure coupling, the states must first be reordered into dependent and independent DOFs with a transformation matrix, $T_{10}$, to compute the reordered state vector $u_{1}$. Following Craig's approach, the constraint equations are applied to the system to generate a new, constrained state vector, $q$. These constraint equations take the form of a matrix multiplication. 


$$
u_{1} \equiv S q
$$

The generalized forms of the mass and stiffness matrices are used in the vehicle dynamics. These matrices are determined by assembling the component mass and stiffness matrices into block-diagonal form $\left(M_{0}, K_{0}\right)$, applying the transformation into independent/dependent coordinates $\left(T_{10}\right)$, and then applying the constraint equations $(S)$. These matrices are further reduced into generalized coordinates for flexible degrees of freedom $(\eta)$ based on the number of modes in the mode shape matrix $(\Phi)$. For simplicity, define the transformation to be:

$$
V \equiv S^{T} T_{10} \Phi
$$

The generalized mass $(\mu)$ and stiffness $(\kappa)$ matrices become

$$
\begin{gathered}
\mu=\Phi^{T} T_{10}^{T} S\left[\begin{array}{ccc}
m_{0}^{\alpha} & & 0 \\
& \ddots & \\
0 & & m_{0}^{\delta}
\end{array}\right] S^{T} T_{10} \Phi=V^{T} M_{0} V \\
\kappa=\Phi^{T} T_{10}^{T} S\left[\begin{array}{ccc}
k_{0}^{\alpha} & & 0 \\
& \ddots & \\
0 & & k_{0}^{\delta}
\end{array}\right] S^{T} T_{10} \Phi=V^{T} K_{0} V
\end{gathered}
$$

Since the mode shapes are held constant, $V$ does not depend on time nor mass properties and can be calculated at initialization. Thus, the generalized mass and stiffness matrices may be calculated directly from the component matrices which are determined by interpolation each frame. Usually one component varies mass for a given time; therefore, Eqs. (16) and (17) are rewritten into a summation that is a function of each component independently. This modification allows the generalized mass and stiffness matrices to be computed once and stored for the constant mass components. A significant reduction in the number of computations is accomplished since the matrix multiplications are on the order of the number of degrees of freedom of one component instead of the entire system.

The separation of $V$ for each component involves partitioning $V$ into submatrices $\left(v_{\alpha}, v_{\beta}, \ldots\right)$ with the same width as the original $V$, but the number of rows of each submatrix is the same as the number of degrees of freedom of the corresponding component.

$$
\begin{gathered}
\mu=\left[\begin{array}{lll}
v_{\alpha}^{T} & \cdots & v_{\delta}^{T}
\end{array}\right]\left[\begin{array}{ccc}
m_{0}^{\alpha} & & 0 \\
& \ddots & \\
0 & & m_{0}^{\delta}
\end{array}\right]\left[\begin{array}{c}
v_{\alpha} \\
\vdots \\
v_{\delta}
\end{array}\right]=\sum_{i} v_{i}^{T} m_{0}^{i} v_{i} \\
\kappa=\left[\begin{array}{lll}
v_{\alpha}^{T} & \cdots & v_{\delta}^{T}
\end{array}\right]\left[\begin{array}{ccc}
k_{0}^{\alpha} & & 0 \\
& \ddots & \\
0 & & k_{0}^{\delta}
\end{array}\right]\left[\begin{array}{c}
v_{\alpha} \\
\vdots \\
v_{\delta}
\end{array}\right]=\sum_{i} v_{i}^{T} k_{0}^{i} v_{i}
\end{gathered}
$$

A simple diagonal damping matrix is created based on a desired damping ratio. The mass integrals are calculated in a similar manner, although detailed derivations of the equations are beyond the scope of this paper.

The matrix multiplications are performed by a special function that implements the Goto/Van de Geijin method ${ }^{5}$, which ensures that the processor is utilizing floating point units while avoiding cache misses. This method uses an inner loop, which iterates over the maximum amount of data that can fit in a processor's level 1 (L1) cache at a time, with outer loops for each higher level of processor cache (L2, L3, etc.).

The individual mass and stiffness matrices for each component must be determined based on stage mass. Lookup tables provide these matrices using the substructure or stage mass as the independent variable. The center of mass and moment of inertia for each stage are determined using the component mass matrix from the geometric rigid 
body modes technique. The center of mass and moment of inertia for the composite vehicle are calculated using the parallel axis theorem and the component mass properties.

\section{B. Multithreading}

The algorithms for looking up the mass and stiffness matrices, calculating mass properties, and solving the equations of motion have been designed to run on multi-core and multi-processor systems. Multi-threading of mass property and matrix calculations is straightforward since the calculations for each stage can be performed on a separate thread. Additionally, the mass integrals can be calculated on a separate thread.

The functions that solve the equations are separated into different tasks, which can be executed in parallel. A function (FApp) sums all the applied forces and moments (including force following terms) about the vehicle frame and calculates the generalized forces and moments. Another function (RHS) calculates the right hand side kinematic terms of Eq. (7). A third function calculates the system mass matrix and performs an LU decomposition. A fourth function performs the forward and backwards substitution (FBS) for the LU decomposed system mass matrix. Figure 4 illustrates a potential multithreading scheme. This scheme is based on using a separate thread for each stage and using separate threads for interpolating components and calculating generalized mass and stiffness matrices.

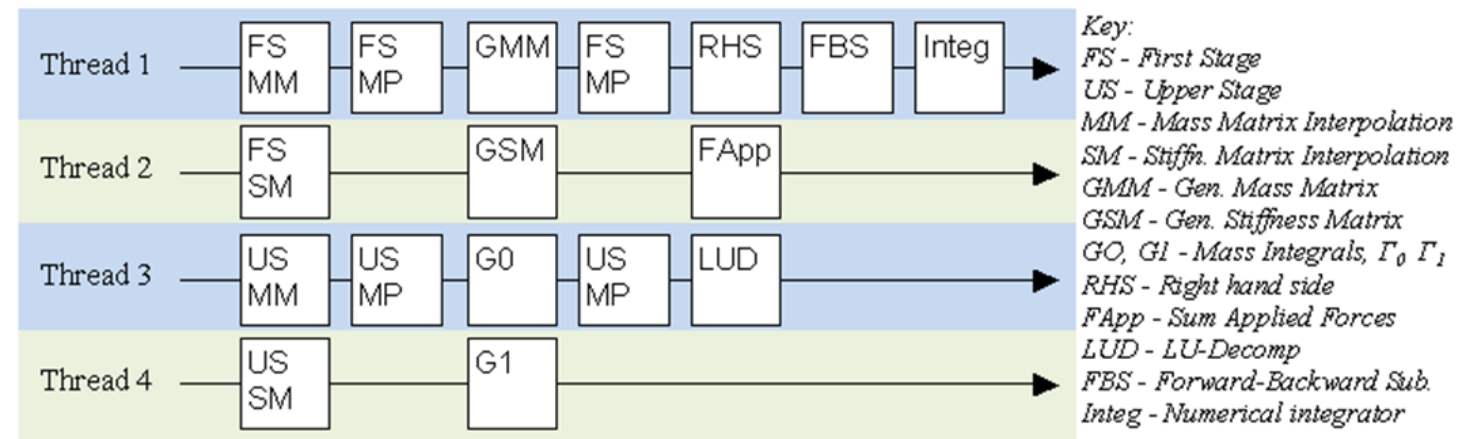

Figure 4. Multithreading of ARTEMIS Dynamics

\section{Example}

To demonstrate the feasibility of this approach, an example problem was formulated and solved with the simulation package TREETOPS. TREETOPS is a non-linear, multibody simulation tool which supports flexible bodies through the assumed modes technique. An example five-element beam, shown in Fig. 5, was constructed in NASTRAN and used as a model of dry structure.

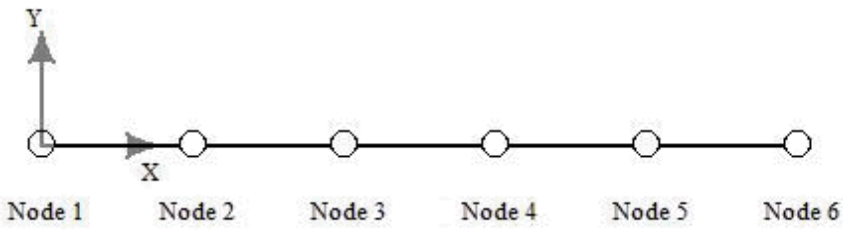

Figure 5. Flexible Beam Structure

Table 1 contains the properties for the dry beam. To simulate propellant, rigid bodies with mass and inertia were connected to each of the six nodes of the beam. The masses were depleted over time using a prescribed flow rate. A TREETOPS model was constructed with seven bodies. Body one was the dry flexible beam. Bodies two through seven represented the propellant nodal masses and inertias rigidly attached to the beam. The structural characteristics of body one were derived from the NASTRAN model of the dry beam. 
Table 1. Beam Properties

\begin{tabular}{|c|c|}
\hline Radius & $0.5 \mathrm{in}$ \\
\hline Length & $10 \mathrm{ft}$ \\
\hline Density & $5.0 \mathrm{slug} / \mathrm{ft}^{3}$ \\
\hline Young's Modulus & $1.44 \times 10^{9} \mathrm{lbf} / \mathrm{ft}^{2}$ \\
\hline Area Moment of Inertia & $2.367 \times 10^{-6} \mathrm{ft}^{4}$ \\
\hline Poisson's ratio & 0.334 \\
\hline Polar Moment of Inertia & $4.735 \times 10^{-6} \mathrm{ft}^{4}$ \\
\hline
\end{tabular}

Table 2 contains the mass properties of each of the rigid bodies attached to the beam nodes representing the full propellant beam configuration. Eleven system NASTRAN models of the beam were created with concentrated masses and inertias attached at each beam node. Each of these models represented a separate mass configuration of the beam during the propellant burn. The propellant burn reduced the masses from the initial value of one slug to zero slugs over a period of fifteen seconds. The inertia of each mass remained unchanged for this example.

Table 2. Propellant Mass Properties

\begin{tabular}{|c|c|}
\hline Mass & 1 slug \\
\hline Ixx & 0.0 slug $\cdot \mathrm{ft}^{2}$ \\
\hline Iyy & 1.0 slug $\cdot \mathrm{ft}^{2}$ \\
\hline Izz & 1.0 slug $\cdot \mathrm{ft}^{2}$ \\
\hline Ixy & 0.0 slug $\cdot \mathrm{ft}^{2}$ \\
\hline Ixz & 0.0 slug $\cdot \mathrm{ft}^{2}$ \\
\hline Iyz & 0.0 slug $\cdot \mathrm{ft}^{2}$ \\
\hline
\end{tabular}

Table 3 contains the first ten frequencies computed in NASTRAN for the final or empty propellant configuration and for the initial or full propellant beam configuration. In this table, the empty propellant configuration consists of the dry beam with attached lumped inertias.

Table 3. Bending Frequency Range for Propellant Depletion

\begin{tabular}{|c|c|c|}
\hline Bending Mode & Empty Propellant & Full Propellant \\
\hline 1 & $3.295551 \mathrm{~Hz}$ & $1.798136 \mathrm{~Hz}$ \\
\hline 2 & $3.295551 \mathrm{~Hz}$ & $1.798136 \mathrm{~Hz}$ \\
\hline 3 & $6.188647 \mathrm{~Hz}$ & $4.327146 \mathrm{~Hz}$ \\
\hline 4 & $6.188647 \mathrm{~Hz}$ & $4.327146 \mathrm{~Hz}$ \\
\hline 5 & $9.068079 \mathrm{~Hz}$ & $7.236610 \mathrm{~Hz}$ \\
\hline 6 & $9.068079 \mathrm{~Hz}$ & $7.236610 \mathrm{~Hz}$ \\
\hline 7 & $11.25814 \mathrm{~Hz}$ & $10.15644 \mathrm{~Hz}$ \\
\hline 8 & $11.25814 \mathrm{~Hz}$ & $10.15644 \mathrm{~Hz}$ \\
\hline 9 & $12.62832 \mathrm{~Hz}$ & $12.27924 \mathrm{~Hz}$ \\
\hline 10 & $12.62832 \mathrm{~Hz}$ & $12.27924 \mathrm{~Hz}$ \\
\hline
\end{tabular}

Typically, the propellant would be simulated in ARTEMIS as a separate structure and then substructure coupled to the dry beam. But for this example, the system was modeled as a single structure. ARTEMIS interpolated directly on the NASTRAN mass matrices of the integrated system. Three sets of system modes were evaluated in ARTEMIS: (A) ten modes from dry beam with lumped inertias (empty configuration), (B) ten modes from dry beam with full masses (full configuration), (C) sixteen combined shapes from ten half full modes, three full modes, and three empty modes. Table 4 lists system frequencies derived from a NASTRAN model of the empty configuration and ARTEMIS frequencies using the various basis vectors. Table 5 lists the same system frequencies for a full system. 
Table 4. Empty System Frequencies

\begin{tabular}{|c|r|r|r|r|}
\hline Mode & $\begin{array}{c}\text { NASTRAN } \\
\text { System } \\
\text { Modes }\end{array}$ & $\begin{array}{c}\text { ARTEMIS } \\
\text { Empty } \\
\text { Modes }\end{array}$ & $\begin{array}{c}\text { ARTEMIS } \\
\text { Full } \\
\text { Modes }\end{array}$ & $\begin{array}{c}\text { (C) } \\
\text { ARTEMIS } \\
\text { Combined } \\
\text { Modes }\end{array}$ \\
\hline 1 & 3.2956 & 3.2956 & 3.3143 & 3.2956 \\
\hline 2 & 3.2956 & 3.2956 & 3.3143 & 3.2956 \\
\hline 3 & 6.1886 & 6.1886 & 6.2775 & 6.1886 \\
\hline 4 & 6.1886 & 6.1886 & 6.2775 & 6.2282 \\
\hline 5 & 9.0681 & 9.0681 & 9.3115 & 9.0681 \\
\hline 6 & 9.0681 & 9.0681 & 9.3115 & 9.0681 \\
\hline 7 & 11.2581 & 11.2581 & 11.5788 & 11.2949 \\
\hline 8 & 11.2581 & 11.2581 & 11.5788 & 11.3658 \\
\hline 9 & 12.6283 & 12.6283 & 12.7900 & 12.6283 \\
\hline 10 & 12.6283 & 12.6283 & 12.7900 & 12.6283 \\
\hline
\end{tabular}

Table 5. Full System Frequencies

\begin{tabular}{|c|c|c|c|c|}
\hline Mode & $\begin{array}{c}\text { NASTRAN } \\
\text { System } \\
\text { Modes }\end{array}$ & $\begin{array}{c}\text { ARTEMIS } \\
\text { Empty } \\
\text { Modes }\end{array}$ & $\begin{array}{c}\text { (B) } \\
\text { ARTEMIS } \\
\text { Full } \\
\text { Modes }\end{array}$ & $\begin{array}{c}\text { (C) } \\
\text { ARTEMIS } \\
\text { Combined } \\
\text { Modes }\end{array}$ \\
\hline 1 & 1.7981 & 1.8114 & 1.7981 & 1.7981 \\
\hline 2 & 1.7981 & 1.8114 & 1.7981 & 1.7981 \\
\hline 3 & 4.3471 & 4.4220 & 4.3471 & 4.3471 \\
\hline 4 & 4.3471 & 4.4220 & 4.3471 & 4.3540 \\
\hline 5 & 7.3266 & 7.5091 & 7.3266 & 7.3266 \\
\hline 6 & 7.3266 & 7.5091 & 7.3266 & 7.3266 \\
\hline 7 & 10.1564 & 10.3638 & 10.1564 & 10.1844 \\
\hline 8 & 10.1564 & 10.3638 & 10.1564 & 10.1934 \\
\hline 9 & 12.2792 & 12.3725 & 12.2792 & 12.2792 \\
\hline 10 & 12.2792 & 12.3725 & 12.2792 & 12.2792 \\
\hline
\end{tabular}

As seen in Tables 4 and 5, no single mode basis set works well for the full spectrum of propellant levels. However, at the computational expense of additional shapes, the combined set of vectors from various mass configurations performs well. Any screening or shape selection process should, as a minimum, evaluate the predicted system frequencies for the configurations being studied. Resulting system mode shapes also need to be considered.

The simulations began with the system at rest. A 500-pound excitation force was applied along the beam Y-axis at node 1 for 0.004 seconds and then released. The simulation was run for constant mass and variable mass problems. Figure 6 depicts the angular velocity of node 1, including bending, and Fig. 7 contains the lateral deflection of node 6 for the full constant mass run. The results show excellent agreement between TREETOPS and ARTEMIS while using different dynamic formulations and support the verification of Eq. 7. Next, a variable mass case was run to evaluate the feasibility of the approach utilized in ARTEMIS and outlined in this paper. A 15 second burn with a burn rate of $1 / 15 \mathrm{slugs} / \mathrm{second}$ for each propellant mass started at 2 seconds. At the end of the burn, the propellant masses were completely depleted. The inertia of each of the propellant masses was not changed for this problem. Figure 8 is the angular velocity of node 1 and Fig. 9 is the lateral deflection of node 1. Again, there is excellent agreement between TREETOPS and ARTEMIS. 


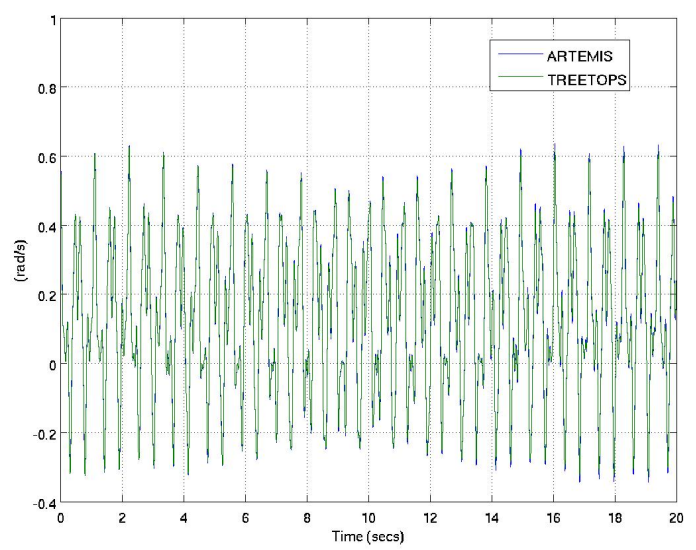

Figure 6. Constant Mass, Node 1 Angular Velocity

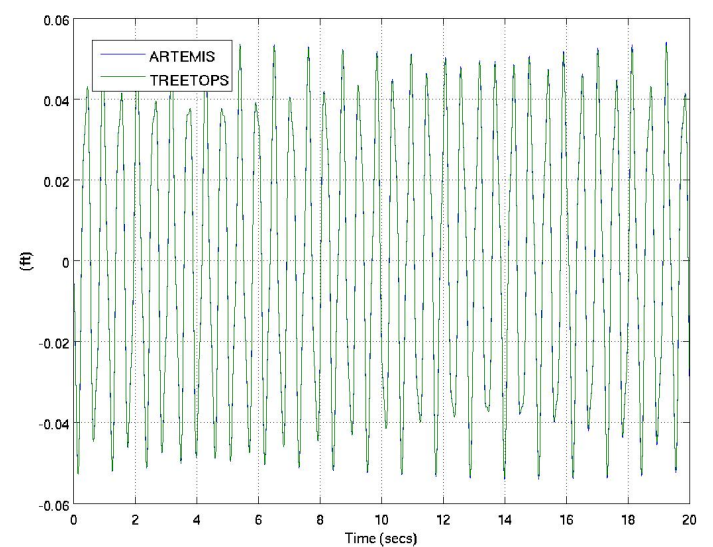

Figure 7. Constant Mass, Node 6 Lateral Deflection

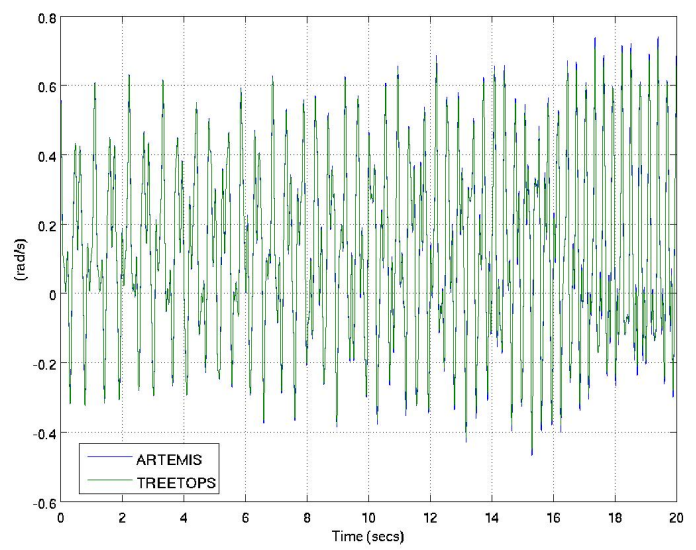

Figure 8. Variable Mass, Node 1 Angular Velocity 


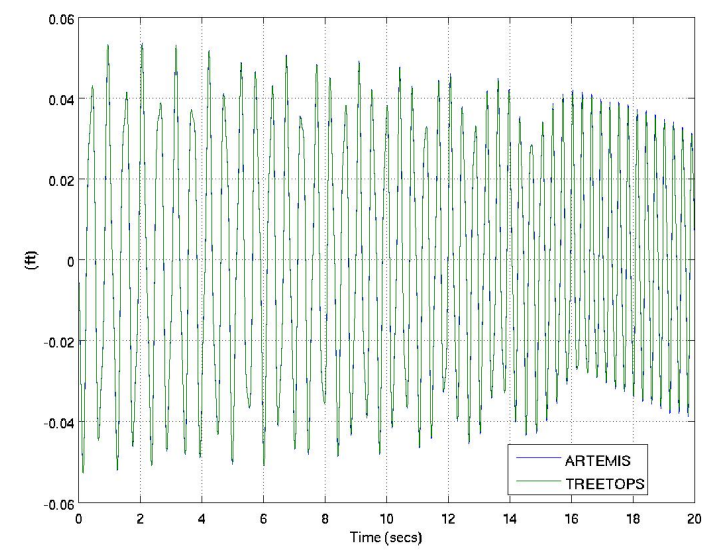

Figure 9. Variable Mass, Node 6 Lateral Deflection

\section{Conclusion}

An approach to efficiently simulate variable mass, flexible body systems has been presented and demonstrated. In this method, shape functions are held constant over a wide range of mass configurations, allowing the simulation tool to utilize existing formulations for the equations of motion of a single flexible body. This technique does not employ time-varying shape functions, which are difficult to characterize and are computationally intensive. This approach also does not utilize scheduled or interpolated shape functions that can introduce numerical discontinuities into the solution. Instead, this method operates directly on substructure mass matrices and updates the system mass matrix, mass properties, and coupling terms in the equations of motion. The Ares I simulation tool ARTEMIS has adopted this technique and expanded the formulation to real-time operation through selective partitioning of the equations and parallel computations. Future work will focus on developing methods to select system modes, evaluating different types of system modes, and reducing numerical discontinuities that can occur during vehicle staging events.

\section{Acknowledgments}

The ARTEMIS development team would like to thank all of the ES50 Flight \& Ground Software Division at MSFC, notably ARTEMIS Lead for NASA Bobby Powell and SIL Team Lead Drew Hall for their support in the development of this technique and the ARTEMIS simulation tool.

\section{References}

${ }^{1}$ Tobbe, P. A., “An Application of Component Modes to the Flexible Multibody Simulation Problem,” Ph.D. Dissertation, Dept. of Mechanical and Aerospace Engineering, Univ. of Alabama in Huntsville, Huntsville, AL, 1995.

${ }^{2}$ Wilson, H. E., "Dynamics of Variable Mass Flexible Bodies with Time Varying Mode Shapes." M.S. Thesis, Dept. of Mechanical and Aerospace Engineering, Univ. of Alabama in Huntsville, Huntsville, AL, 2007.

${ }^{3}$ User's Manual for TREETOPS, Rev. 8, Dynacs Inc, Clearwater, FL, 1990.

${ }^{4}$ Craig, R. R., Structural Dynamics: An Introduction to Computer Methods, John Wiley \& Sons, New York, 1981.

${ }^{5}$ Goto, K., van de Geijn, R. A., "Anatomy of High-Performance Matrix Multiplication," ACM Transactions on Mathematical Software, Vol. 34, No. 3, 2008, Article 12. 
Modeling and Simulation of Variable

\section{Mass, Flexible Structures}

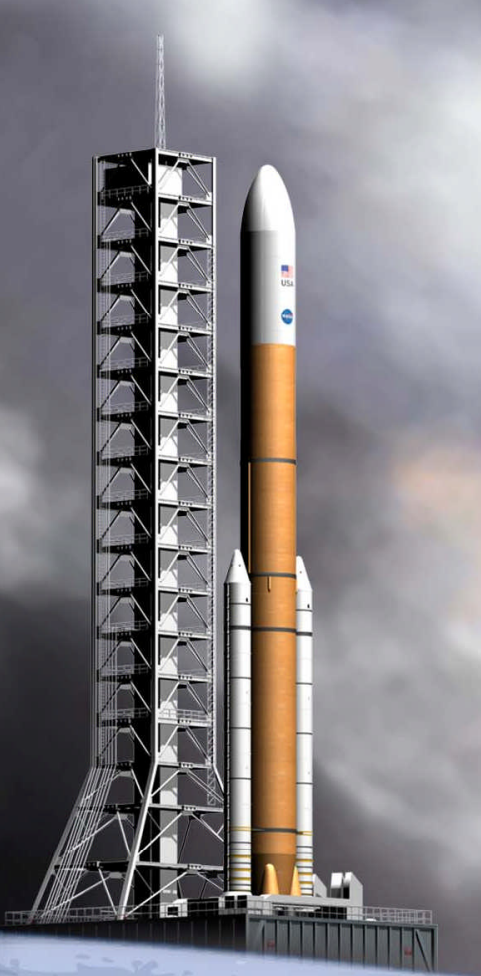

Patrick Tobbe, Ph. D. Alex Matras, Ph. D. Heath Wilson Dynamic Concepts, Inc. Huntsville, Ala. 


\section{Outline}

- Equations of Motion of a Flexible Body

- Variable Mass Challenge and Solutions

- ARTEMIS Simulation Description

- Implementation of Equations of Motion

- Initial Validation Test Case

- Results / Observations

- Future Work 
- Formulation to accurately simulate the response of a single vehicle

- Derived using modified form of Boltzmann-Hamel equations

- Energy method allowing kinetic and potential energies to be expressed in coordinates in rotating frame

- Structural flexibility of vehicles / bodies modeled using assumed modes technique

- Equations of motion allow for large rigid body translations and rotations coupled with small body deformations 


\section{Boltzman-Hamel Method}

\section{Boltzmann-Hamel Equations}

$\frac{d}{d t}\left(\frac{\partial T}{\partial \dot{\mathbf{R}}^{B}}\right)+\boldsymbol{\omega}^{B} \times \frac{\partial T}{\partial \dot{\mathbf{R}}^{B}}-[B I] \frac{\partial T}{\partial \mathbf{R}^{I}}+[B I] \frac{\partial V^{*}}{\partial \mathbf{R}^{I}}=[B I] \mathbf{Q}_{R^{I}}$

$\frac{d}{d t}\left(\frac{\partial T}{\partial \boldsymbol{\omega}^{B}}\right)+\boldsymbol{\omega}^{B} \times \frac{\partial T}{\partial \boldsymbol{\omega}^{B}}+\dot{\mathbf{R}}^{B} \times$
$\frac{d}{d t}\left(\frac{\partial T}{\partial \dot{\boldsymbol{\eta}}}\right)-\frac{\partial T}{\partial \boldsymbol{\eta}}+\frac{\partial V^{*}}{\partial \boldsymbol{\eta}}=\mathbf{Q}_{\eta}$

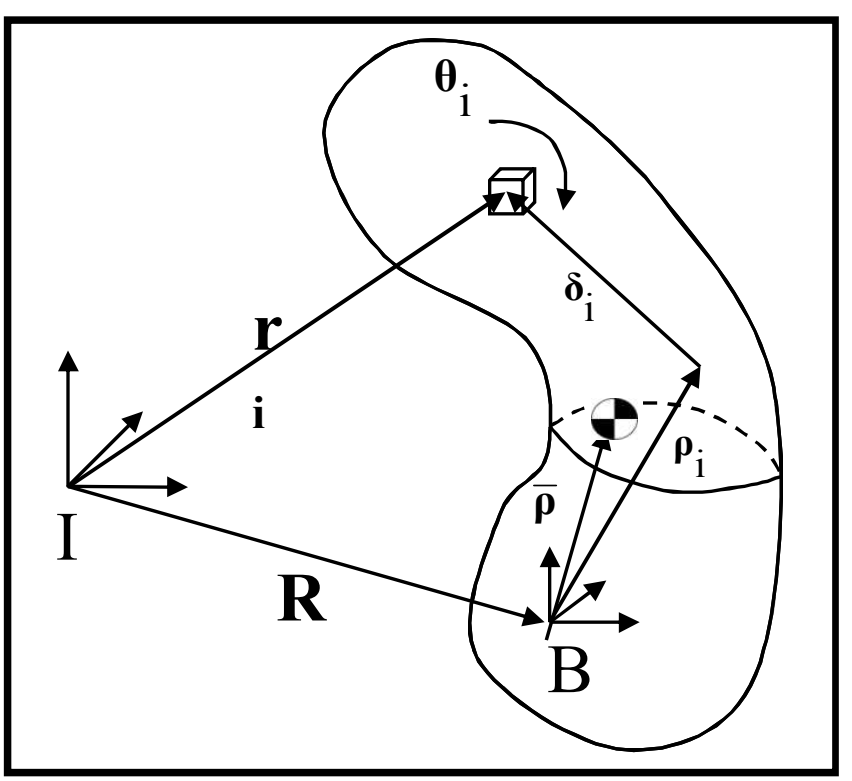

Kinematics

$\mathbf{r}_{i}=\mathbf{R}+\boldsymbol{\rho}_{i}+\boldsymbol{\delta}_{i}$

$\dot{\mathbf{r}}_{i}=\dot{\mathbf{R}}+\boldsymbol{\omega}^{B} \times\left(\boldsymbol{\rho}_{i}+\boldsymbol{\delta}_{i}\right)+\dot{\boldsymbol{\delta}}_{i}$

$\boldsymbol{\delta}_{i}=\boldsymbol{\varphi}_{i j} \eta_{j}$

$\dot{\boldsymbol{\delta}}_{i}=\boldsymbol{\varphi}_{i j} \dot{\eta}_{j}$

$\boldsymbol{\theta}_{i}=\boldsymbol{\psi}_{i j} \eta_{j}$

$\dot{\boldsymbol{\theta}}_{i}=\boldsymbol{\psi}_{i j} \dot{\eta}_{j}$

Energy Formulations

$$
\begin{aligned}
T & =\sum_{i}\left(\frac{1}{2} m_{i}\left(\dot{\mathbf{r}}_{\mathbf{i}} \cdot \dot{\mathbf{r}}_{\mathbf{i}}\right)+\frac{1}{2}\left(\boldsymbol{\omega}^{B}+\dot{\boldsymbol{\theta}}_{i}\right)^{T} \mathbf{I}_{i}\left(\boldsymbol{\omega}^{B}+\dot{\boldsymbol{\theta}}_{i}\right)\right) \\
V^{*} & =\frac{1}{2}\left[\begin{array}{l}
\boldsymbol{\delta} \\
\boldsymbol{\theta}
\end{array}\right]^{T} \mathbf{K}\left[\begin{array}{l}
\boldsymbol{\delta} \\
\boldsymbol{\theta}
\end{array}\right]
\end{aligned}
$$




\section{Equations of Motion}

$$
\begin{aligned}
& {\left[\begin{array}{ccc}
\frac{m \mathbf{I}_{3 x 3}}{\widetilde{\boldsymbol{\Gamma}_{0} \boldsymbol{\eta}+m \overline{\boldsymbol{\rho}}}} & -\left(\widetilde{\left.\boldsymbol{\Gamma}_{0} \boldsymbol{\eta}+m \overline{\boldsymbol{\rho}}\right)}\right. & \boldsymbol{\Gamma}_{0} \\
\boldsymbol{\Gamma}_{0}^{T} & \left.\mathbf{I}_{1 j}+\mathbf{I}_{1 j}^{T}\right) \eta_{j}+\frac{1}{2}\left(\mathbf{I}_{2 j k}+\mathbf{I}_{2 j k}^{T}\right) \eta_{j} \eta_{k} & \boldsymbol{\Gamma}_{1}+\boldsymbol{\Gamma}_{2 j} \\
\boldsymbol{\Gamma}_{1}^{T}+\boldsymbol{\Gamma}_{2 j}^{T} & \boldsymbol{\mu}
\end{array}\right]\left\{\begin{array}{c}
\dot{ }_{\mathbf{R}}^{B} \\
\dot{\boldsymbol{\omega}}^{B} \\
\ddot{\boldsymbol{\eta}}
\end{array}\right\}=} \\
& \mathbf{F}^{B}-\boldsymbol{\omega}^{B} \times\left(m \dot{\mathbf{R}}^{B}+\boldsymbol{\omega}^{B} \times\left(m \overline{\boldsymbol{\rho}}+\boldsymbol{\Gamma}_{0} \boldsymbol{\eta}+\mathbf{2} \boldsymbol{\Gamma}_{0} \dot{\boldsymbol{\eta}}\right)\right) \\
& \mathbf{T}^{B}-\boldsymbol{\omega}^{B} \times\left(\mathbf{I}_{B}+\left(\mathbf{I}_{1 j}+\mathbf{I}_{1 j}^{T}\right) \eta_{j}+\frac{1}{2}\left(\mathbf{I}_{2 j k}+\mathbf{I}_{2 j k}^{T}\right) \eta_{j} \eta_{k}\right) \boldsymbol{\omega}^{B} \\
& -\boldsymbol{\omega}^{B} \times\left(\boldsymbol{\Gamma}_{1} \dot{\boldsymbol{\eta}}+\boldsymbol{\Gamma}_{2 j k} \eta_{j} \dot{\eta}_{k}\right)-\left(\dot{\mathbf{R}}^{B} \times \boldsymbol{\omega}^{B}\right) \times\left(\boldsymbol{\Gamma}_{0} \dot{\boldsymbol{\eta}}+m \overline{\boldsymbol{\rho}}\right) \\
& -\left(\left(\mathbf{I}_{1 j}+\mathbf{I}_{1 j}^{T}\right) \dot{\eta}_{j}+\frac{1}{2}\left(\mathbf{I}_{2 j k}+\mathbf{I}_{2 j k}^{T}\right)\left(\eta_{j} \dot{\eta}_{k}+\dot{\eta}_{j} \eta_{k}\right)\right) \mathbf{\omega}^{B} \\
& \left(\boldsymbol{\varphi}_{i}^{T} F_{i}+\boldsymbol{\psi}_{i}^{T} T_{i}-\boldsymbol{\kappa} \boldsymbol{\eta}+\boldsymbol{\Gamma}_{0}^{T}\left(\boldsymbol{\omega}^{B} \times \dot{\mathbf{R}}^{B}\right)+\frac{1}{2} \boldsymbol{\omega}^{B^{T}}\left(2 \mathbf{I}_{1 j}+\mathbf{I}_{2 j k} \eta_{k}+\mathbf{I}_{2 j k}^{T} \eta_{k}\right) \boldsymbol{\omega}^{B}\right)
\end{aligned}
$$




$$
\begin{aligned}
& \boldsymbol{\Gamma}_{0 j}=\sum_{i} m_{i} \boldsymbol{\varphi}_{i j} \\
& \boldsymbol{\Gamma}_{1 j}=\sum_{i}\left(m_{i} \boldsymbol{\rho}_{i} \times \boldsymbol{\varphi}_{i j}+\mathbf{I}_{i} \boldsymbol{\psi}_{i j}\right) \\
& \boldsymbol{\Gamma}_{2 j k}=\sum_{i}\left(m_{i} \boldsymbol{\varphi}_{i j} \times \boldsymbol{\varphi}_{i k}\right)
\end{aligned}
$$$$
\begin{aligned}
& \mathbf{I}_{1 j}=\sum_{i} m_{i}\left(\boldsymbol{\rho}_{i} \boldsymbol{\varphi}_{i j} \mathbf{I}_{3 x 3}-\boldsymbol{\varphi}_{i j} \boldsymbol{\rho}_{i}^{T}\right) \\
& \mathbf{I}_{2 j k}=\sum_{i} m_{i}\left(\boldsymbol{\rho}_{i} \boldsymbol{\varphi}_{i j}^{T} \mathbf{I}_{3 x 3}-\boldsymbol{\varphi}_{i j} \boldsymbol{\varphi}_{i k}^{T}\right)
\end{aligned}
$$

- Mass integrals model coupling between rigid body and flex body dynamics

- Using momentum arguments, developed ways of calculating these from mass matrices

- $\Gamma_{0}$ and $\Gamma_{1}$ go to zero when mode shapes are orthogonal to mass matrix, the others are negligible

- Not always the case using our approach

- The $\Gamma_{2}, I_{1} \& I_{2}$ mass integrals may be neglected for systems that do not undergo very large deformations (e.g. for the Ares I) 


\section{Equations of Motion}

Flex Body Eqs. of Motion

$\left[\begin{array}{ccc}\frac{m \mathbf{I}_{3 \times 3}}{\boldsymbol{\Gamma}_{0} \boldsymbol{\eta}+m \overline{\boldsymbol{\rho}}} & -\left(\widetilde{\boldsymbol{\Gamma}_{0} \boldsymbol{\eta}+m \overline{\bar{\rho}}}\right) & \boldsymbol{\Gamma}_{0} \\ \boldsymbol{\Gamma}_{\mathbf{0}}^{T} & \mathbf{I}_{B} & \boldsymbol{\Gamma}_{1} \\ \boldsymbol{\Gamma}_{1}^{T} & \boldsymbol{\mu}\end{array}\right]\left\{\begin{array}{c}\dot{\mathbf{R}}^{B} \\ \dot{\boldsymbol{\omega}}^{B} \\ \ddot{\boldsymbol{\eta}}\end{array}\right\}=$

$\left\{\begin{array}{c}\mathbf{F}^{B}-\boldsymbol{\omega}^{B} \times\left(m \dot{\mathbf{R}}^{B}+\boldsymbol{\omega}^{B} \times\left(m \overline{\boldsymbol{\rho}}+\boldsymbol{\Gamma}_{0} \boldsymbol{\eta}+\mathbf{2} \boldsymbol{\Gamma}_{0} \dot{\boldsymbol{\eta}}\right)\right) \\ \mathbf{T}^{B}-\boldsymbol{\omega}^{B} \times \mathbf{I}_{B} \boldsymbol{\omega}^{B}-\boldsymbol{\omega}^{B} \times \boldsymbol{\Gamma}_{1} \dot{\boldsymbol{\eta}}-\left(\dot{\mathbf{R}}^{B} \times \boldsymbol{\omega}^{B}\right) \times\left(\boldsymbol{\Gamma}_{0} \dot{\boldsymbol{\eta}}+m \overline{\boldsymbol{\rho}}\right) \\ \boldsymbol{\varphi}_{i}^{T} F_{i}+\boldsymbol{\psi}_{i}^{T} T_{i}-\mathbf{\kappa} \boldsymbol{\eta}+\boldsymbol{\Gamma}_{0}^{T}\left(\boldsymbol{\omega}^{B} \times \dot{\mathbf{R}}^{B}\right)\end{array}\right\}$

$\dot{\mathbf{R}}^{I}=\mathbf{T}_{B I} \dot{\mathbf{R}}^{B}$

$\dot{\mathbf{q}}^{B I}=\mathbf{E} \dot{\boldsymbol{\omega}}^{B}$

Modal Reduction

$\boldsymbol{\mu}=\boldsymbol{\Phi}^{T} \mathbf{M}_{1} \boldsymbol{\Phi}$

$\boldsymbol{\kappa}=\boldsymbol{\Phi}^{T} \mathbf{K}_{1} \boldsymbol{\Phi}$

Notation

$\widetilde{\mathbf{x}}=\left[\begin{array}{ccc}0 & -x_{3} & x_{2} \\ x_{3} & 0 & -x_{1} \\ -x_{2} & x_{1} & 0\end{array}\right]$ $\mathrm{m}$ - Total vehicle mass

$\mathrm{I}_{\mathrm{B}}$ - Total vehicle $\mathrm{MOI}$

$\bar{\rho}-$ Center of mass

$\mathrm{F}^{\mathrm{B}}$ - Sum of forces

$T^{\mathrm{B}}-$ Sum of torques

$\phi-$ Trans. mode shapes

$\psi$ - Rot. mode shapes

$\Phi$ - Mode shape matrix

$\mathrm{M}_{1}$ - Total veh. mass matrix

$\mathrm{K}_{1}$ - Total veh. stiff matrix

State vector:

$R^{\prime}-$ Pos. in inertial space

$\dot{R}^{B}-$ Vel. in body frame

$q^{B I}$ - Quaternions

$\omega^{B}$ - Ang. vel. in body frame

$\eta$-Flex states

State derivative vector:

$\dot{\mathrm{R}}^{\prime}$ - Total vel. in inertial space

$\stackrel{\circ}{\mathrm{R}}^{B}-$ Acc. in body frame

$\dot{\mathrm{q}}^{\mathrm{Bl}}$ - Quaternion derivatives

$\dot{\omega}^{\mathrm{B}}$ - Ang. acc. in body frame

$\dot{\eta}$ - Flex state derivatives 7 
- For variable mass systems, such as the Ares I launch vehicle, system mode shapes are not constant

- Depending on structure, system stiffness characteristics could also vary with mass

- Current flexible body simulation tools are not configured for this class of systems

- Mass and stiffness must be determined each step

- Generalized mass and stiffness and mass integrals must be recomputed (large matrices, very time consuming) 


\section{Variable Mass Solutions}

- Schedule / interpolate new system shapes as a function of mass

- Discontinuities in deformations at updates

- Orthogonality of modes with respect to system mass and stiffness matrices

- Determining the rate to update modal data

- Add time varying mode shape terms to derivation of EOM (Wilson)

- Additional terms add significant computation time

- Computing derivatives of mode shapes for vehicle configurations is very difficult

- Hold shape functions constant between staging and vary physical (FEM) mass and stiffness matrix (i.e. use Ritz vectors)

- Partition matrix equations by component, and only perform calculations on parts which change 


\section{Substructure Coupling}

- Method of applying constraints in order to assemble multiple mass matrices into one composite matrix (Craig)

- Select dependent degrees of freedom to be constrained out

- Reorder degrees of freedom to separate dependent DOFs out

- Used to assemble and partition generalized vehicle mass and stiffness matrix calculations

Block diagonal of comp. $m \& k$

Composite stack M \& K

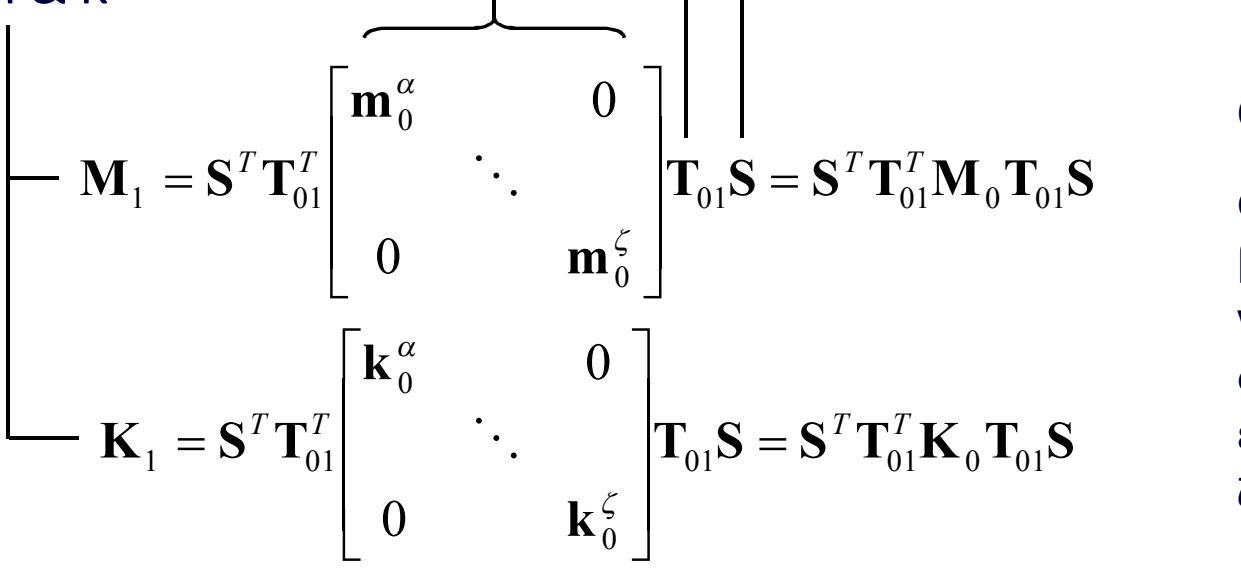

Components

$\alpha$ - Crew module

$\beta$-Service module

$\gamma$ - Upper stage

$\delta$ - Interstage

$\varepsilon-$ First stage

$\zeta$ - Launch abort system 


\section{Partitioning of Calculations}

- Gen. mass matrix as function of comp. mass matrices

$$
\begin{gathered}
\boldsymbol{\mu}=\boldsymbol{\Phi}^{T} \mathbf{S}^{T} \mathbf{T}_{01}^{T}\left[\begin{array}{ccc}
\mathbf{m}_{0}^{\alpha} & & 0 \\
& \ddots & \\
0 & & \mathbf{m}_{0}^{\delta}
\end{array}\right] \mathbf{T}_{01} \mathbf{S} \boldsymbol{\Phi} \\
\boldsymbol{\mu}=\mathbf{V}^{T} \mathbf{M}_{0} \mathbf{V}
\end{gathered}
$$

- Partition by component

- Only components whose mass properties change need to be recalculated

- Calculations can be done in parallel on multi-processor computers

- $v_{i}$ can be calculated at initialization

$$
\begin{gathered}
\mathbf{V} \equiv\left\lfloor\begin{array}{llll}
\mathbf{v}_{\alpha} & \mathbf{v}_{\beta} & \cdots & \mathbf{v}_{\zeta}
\end{array}\right\rfloor \\
\boldsymbol{\mu}=\mathbf{v}_{\alpha} \mathbf{m}_{0}^{\alpha} \mathbf{v}_{\alpha}^{T}+\mathbf{v}_{\beta} \mathbf{m}_{0}^{\beta} \mathbf{v}_{\beta}^{T}+\cdots+\mathbf{v}_{\zeta} \mathbf{m}_{0}^{\zeta} \mathbf{v}_{\zeta}^{T}
\end{gathered}
$$

- Generalized stiffness matrix and mass integrals are derived into an analogous form 
- Ares Real Time Environment for Modeling, Simulation and Integration (ARTEMIS) is the real time simulation of Ares I supporting the development and validation of Ares I flight software and avionics components

- Accurately models all Ares I/Orion/Ground systems which interact with Ares I avionics components from pre-launch through orbit insertion

- Interacts with lab configuration and control software to support model selection and fault insertion

- Operates in a hardware-in-the-loop environment such that a user can select between software models of avionics components or interface to actual avionics hardware

- Simulated models must communicate with actual hardware via MIL-STD1553B, Gigabit ethernet, RS-422, discrete and analog signals

- Includes models of vehicle dynamics, environment and subsystem models

- Must maintain hard real-time operation

- Uses very specialized functions for matrix multiplication (see Goto \& van de Geijn) for optimal performance 


\section{Example Lab Configurations}

MAESTRO Ethernet

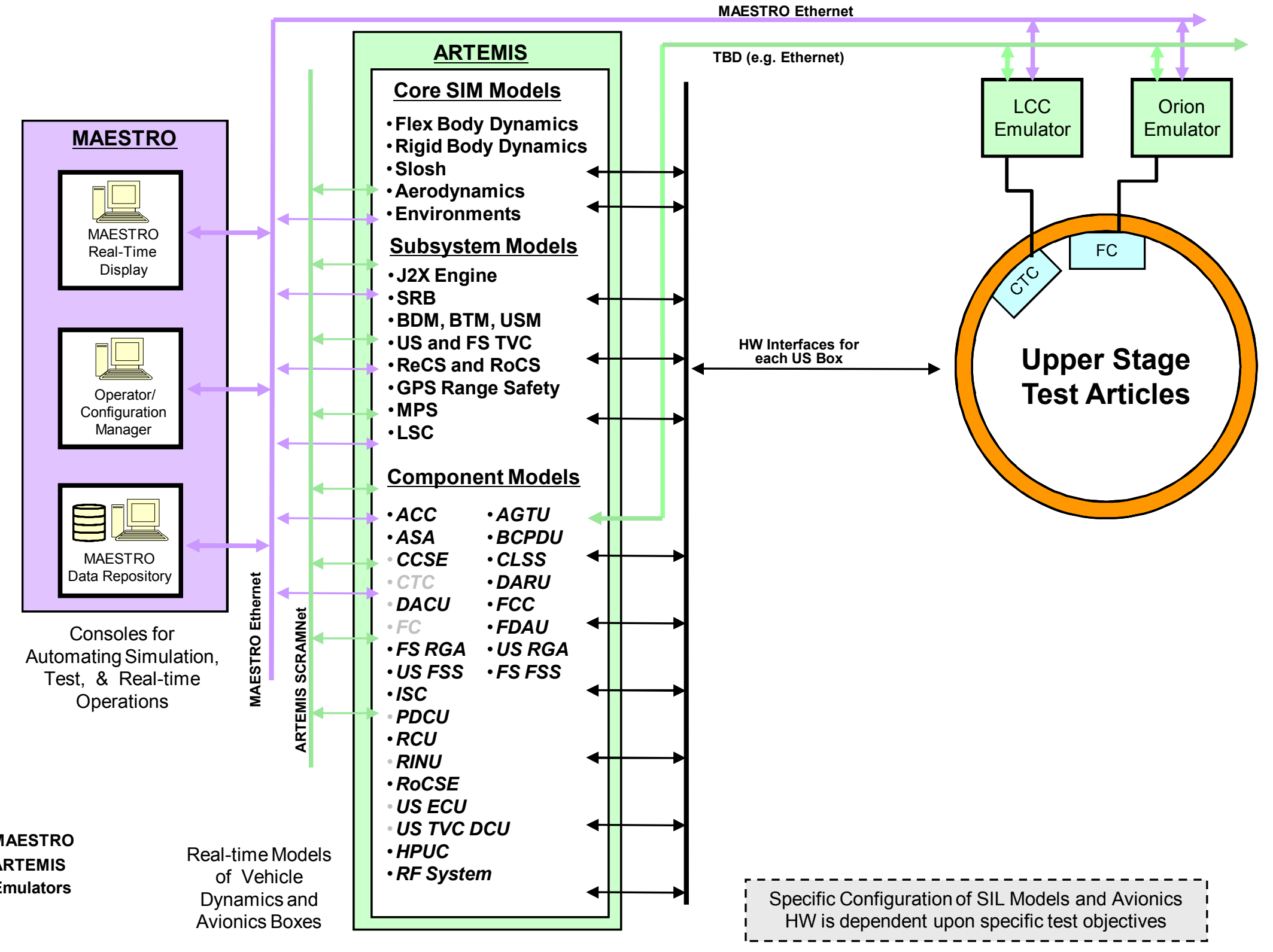




\section{Example Lab Configurations}

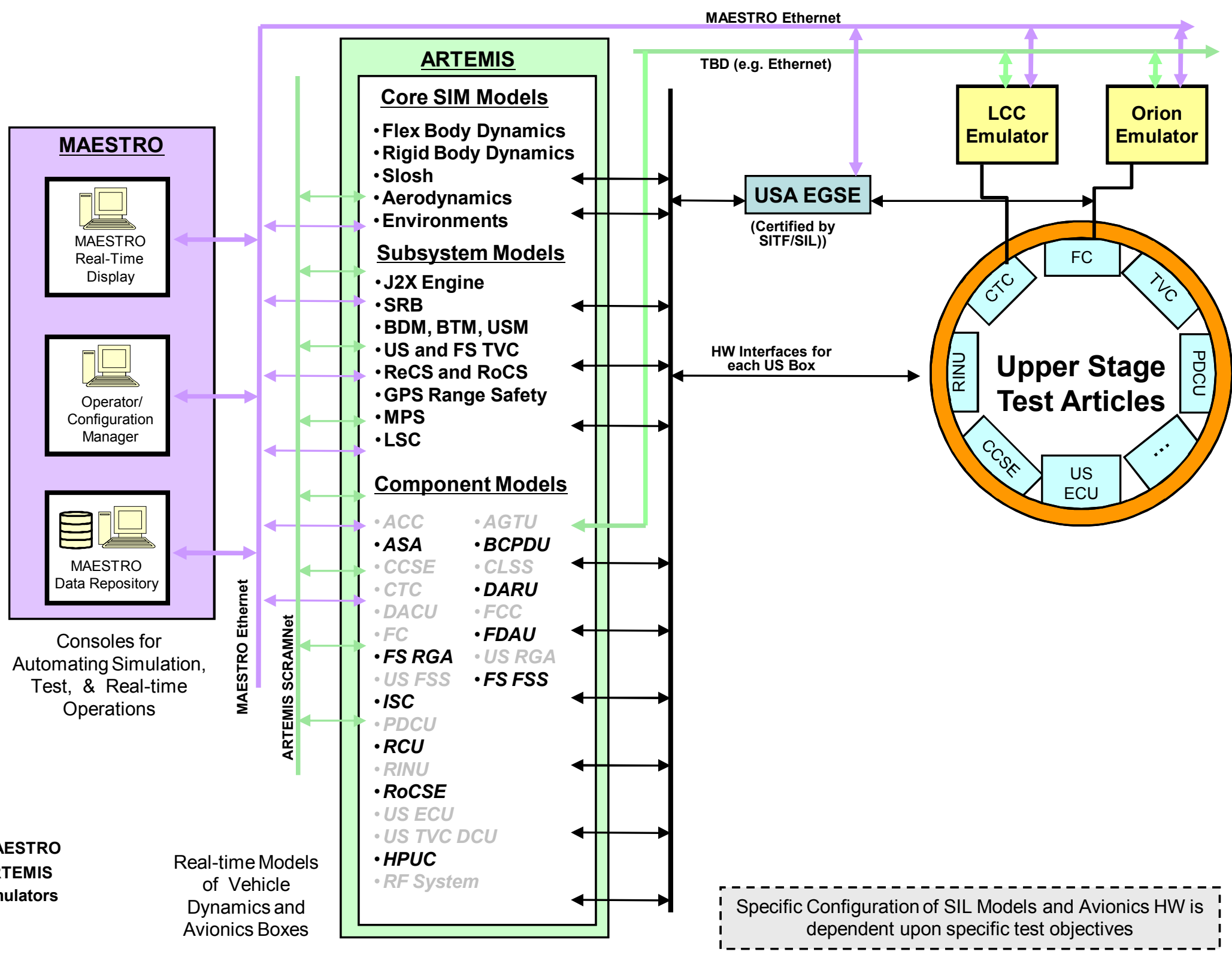




\section{ARTEMIS Flexible Body Configuration}

- Define vehicle as a single body composed of components (stages) connected at nodes

- Each component is composed of structure and propellant

- Component input data consists of FEM mass and stiffness matrices, DOF map, and node geometry of structure and propellant

- Re-compute propellant mass and stiffness matrices each integration time step via interpolation with respect to mass

- Update component mass properties using geometric rigid body modes technique

- Assemble vehicle mass and stiffness matrices each integration time step via automated substructure coupling

- Use linearly independent combination of vehicle mode shapes / Ritz vectors computed from various mass configurations analyzed

- Hold mode shapes or Ritz vectors constant

- At staging event, update vehicle geometry, mass properties, Ritz vectors

- Spawn and propagate separated rigid vehicle (can be composed of multiple cores or stages) based on flight phase 


\section{Ares I Flight Phases}

LAS

Crew Module

Service Module

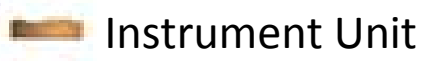

A US

J2-X

$\mathrm{A}$

Innerstage

FS

SRB

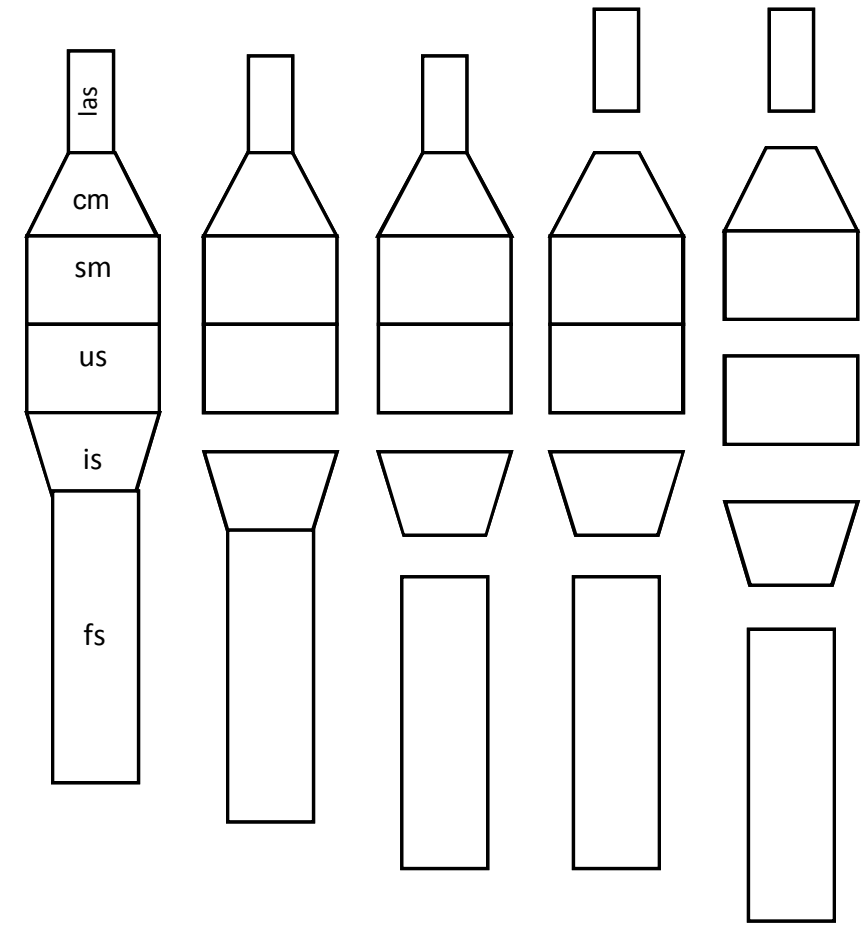

Nominal Flight Phases
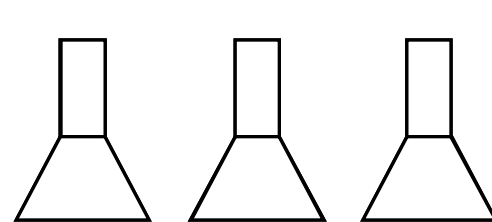

$\square$
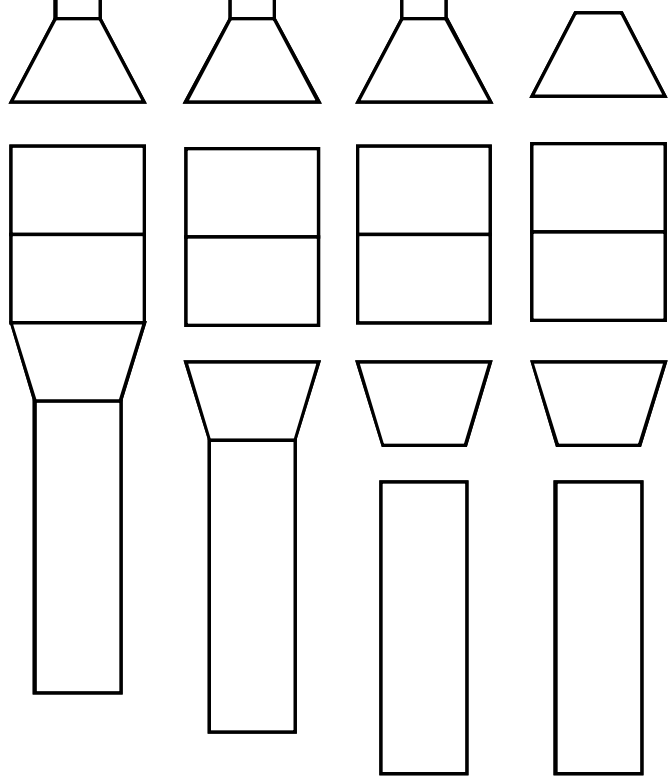

Abort Phases 


\section{Variable Mass, Flexible Body Test Case}

- Developed variable mass, flexible body example problem composed of dry beam structure augmented with propellant nodes

- Applied sinusoidal force to end node of beam while depleting mass from propellant nodes

- No modal damping

- Constructed 36 DOF FEM of dry beam

- Developed FEMs of dry beam and various levels of propellant

- Extracted mass and stiffness matrices from FEM for various levels of propellant for use in ARTEMIS

- Computed system frequencies for empty and full propellant levels to support Ritz vector selection in ARTEMIS

- Serves as validation for the implementation of flex body dynamics in ARTEMIS 


\section{Test Case}

- Sinusoidal force applied in -Y direction at node 1 from time $=0$ for 0.4 seconds with a magnitude of 10 pounds

- Mass depletion rate of $1 / 15$ slugs/second starting at time $=2$ seconds until time $=17$ seconds

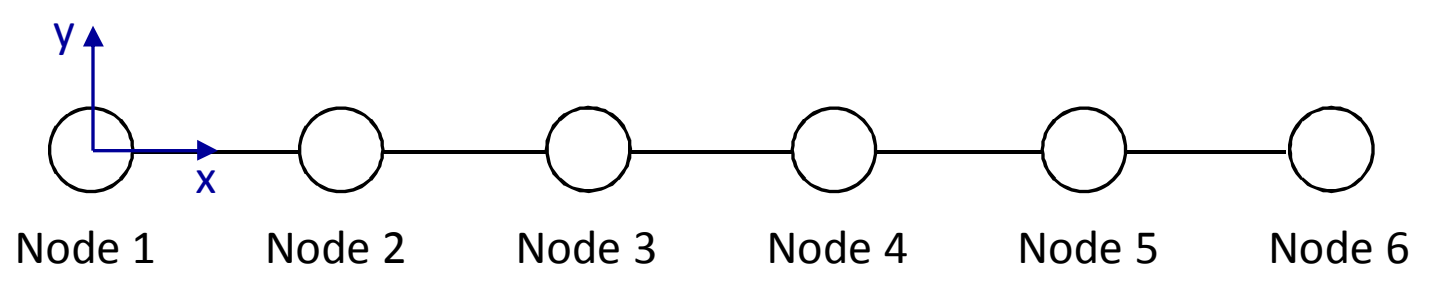


- Configured ARTEMIS for a single, flexible body using constant set of system modes and library of system mass and stiffness matrices

- Used three different sets of shapes in ARTEMIS : empty system, full system, and combination of empty and full systems

- Computed system frequencies from ARTEMIS M and $\mathrm{K}$ for empty and full propellant levels, compared to system FEM modes

- Applied prescribed forcing function to node 1

- Computed total angular velocity of node 1 and translational deflection of node 6 for constant mass and variable mass configurations 


\section{Test Case}

\begin{tabular}{|c|c|c|c|}
\hline Radius & $0.5 \mathrm{in}$ & Mass & $1.0 \mathrm{slug}$ \\
\hline Length & $10 \mathrm{ft}$ & $\mathrm{I}_{\mathrm{xx}}$ & $0.0 \mathrm{slug} \cdot \mathrm{ft}^{2}$ \\
\hline Density & $5.0 \mathrm{slug} / \mathrm{ft}^{3}$ & $\mathrm{I}_{\mathrm{yy}}$ & $1.0 \mathrm{slug} \cdot \mathrm{ft}^{2}$ \\
\hline $\begin{array}{c}\text { Young's } \\
\text { Modulus }\end{array}$ & $\begin{array}{c}1.44 \times 10^{9} \\
\mathrm{lbf} / \mathrm{ft}^{2}\end{array}$ & $\mathrm{I}_{\mathrm{zz}}$ & $1.0 \mathrm{slug} \cdot \mathrm{ft}^{2}$ \\
\hline $\begin{array}{c}\text { Area Moment } \\
\text { of Inertia }\end{array}$ & $2.367 \times 10^{-6} \mathrm{ft}^{4}$ & $\mathrm{I}_{\mathrm{xy}}$ & $0.0 \mathrm{slug} \cdot \mathrm{ft}^{2}$ \\
\hline \begin{tabular}{c} 
Poisson's ratio \\
\hline $\begin{array}{c}\text { Polar Moment } \\
\text { of Inertia }\end{array}$
\end{tabular} & 0.334 & $\mathrm{I}_{\mathrm{xz}}$ & $0.0 \mathrm{slug} \cdot \mathrm{ft}^{2}$ \\
\hline
\end{tabular}




\section{FEM System Natural Frequencies}

\begin{tabular}{|c|c|c|}
\hline Bending Mode & Empty Propellant & Full Propellant \\
\hline 1 & $3.295551 \mathrm{~Hz}$ & $1.798136 \mathrm{~Hz}$ \\
\hline 2 & $3.295551 \mathrm{~Hz}$ & $1.798136 \mathrm{~Hz}$ \\
\hline 3 & $6.188647 \mathrm{~Hz}$ & $4.327146 \mathrm{~Hz}$ \\
\hline 4 & $6.188647 \mathrm{~Hz}$ & $4.327146 \mathrm{~Hz}$ \\
\hline 5 & $9.068079 \mathrm{~Hz}$ & $7.236610 \mathrm{~Hz}$ \\
\hline 6 & $9.068079 \mathrm{~Hz}$ & $7.236610 \mathrm{~Hz}$ \\
\hline 7 & $11.25814 \mathrm{~Hz}$ & $10.15644 \mathrm{~Hz}$ \\
\hline 8 & $11.25814 \mathrm{~Hz}$ & $10.15644 \mathrm{~Hz}$ \\
\hline 9 & $12.62832 \mathrm{~Hz}$ & $12.27924 \mathrm{~Hz}$ \\
\hline 10 & $12.62832 \mathrm{~Hz}$ & $12.27924 \mathrm{~Hz}$ \\
\hline
\end{tabular}




\section{Full Propellant Configuration}

\begin{tabular}{|c|r|r|r|r|}
\hline Mode & \multicolumn{1}{|c|}{$\begin{array}{c}\text { NASTRAN } \\
\text { System Modes }\end{array}$} & $\begin{array}{c}\text { (A) ARTEMIS Empty } \\
\text { Modes Basis }\end{array}$ & $\begin{array}{c}\text { (C) ARTEMIS ARTEMIS } \\
\text { Full Modes Basis }\end{array}$ & $\begin{array}{c}\text { Combined Modes } \\
\text { Basis }\end{array}$ \\
\hline 1 & 1.7981 & 1.8114 & 1.7981 & 1.7981 \\
\hline 2 & 1.7981 & 1.8114 & 1.7981 & 1.7981 \\
\hline 3 & 4.3471 & 4.4220 & 4.3471 & 4.3471 \\
\hline 4 & 4.3471 & 4.4220 & 4.3471 & 4.3540 \\
\hline 5 & 7.3266 & 7.5091 & 7.3266 & 7.3266 \\
\hline 6 & 7.3266 & 7.5091 & 7.3266 & 7.3266 \\
\hline 7 & 10.1564 & 10.3638 & 10.1564 & 10.1844 \\
\hline 8 & 10.1564 & 10.3638 & 10.1564 & 10.1934 \\
\hline 9 & 12.2792 & 12.3725 & 12.2792 & 12.2792 \\
\hline 10 & 12.2792 & 12.3725 & 12.2792 & 12.2792 \\
\hline
\end{tabular}


- TREETOPS is a multibody simulation package developed at the Marshall Spaceflight Center that supports flexible bodies

- Built TREETOPS simulation of dry flexible beam (body 1) rigidly attached to propellant masses (bodies 2 through 7)

- Described structural flexibility of body 1 through dry beam modes

- Applied prescribed forcing function to node 1

- Computed total angular velocity of node 1 and translational deflection of node 6 for constant mass and variable mass configurations 


\section{Simulation Results}

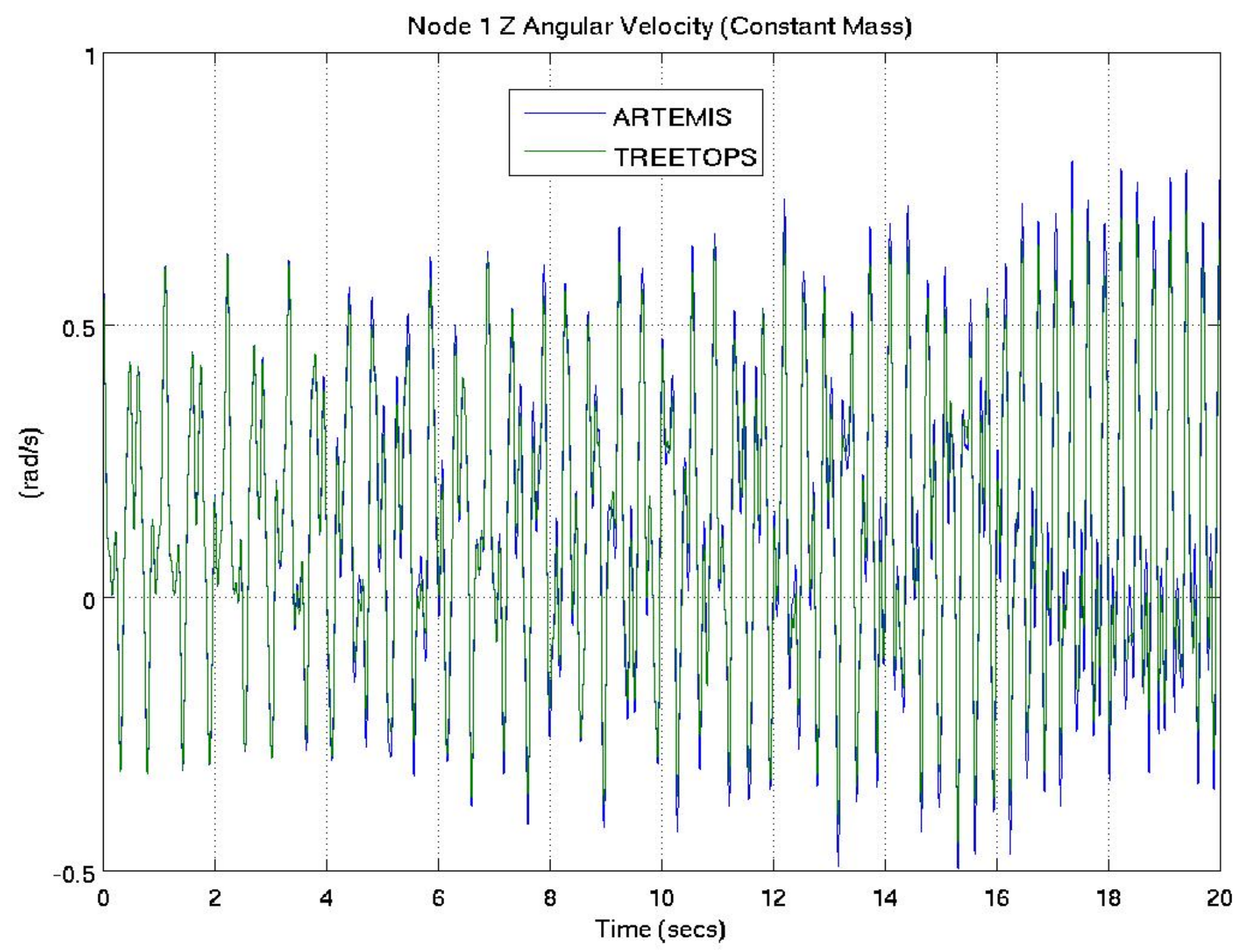




\section{Simulation Results}

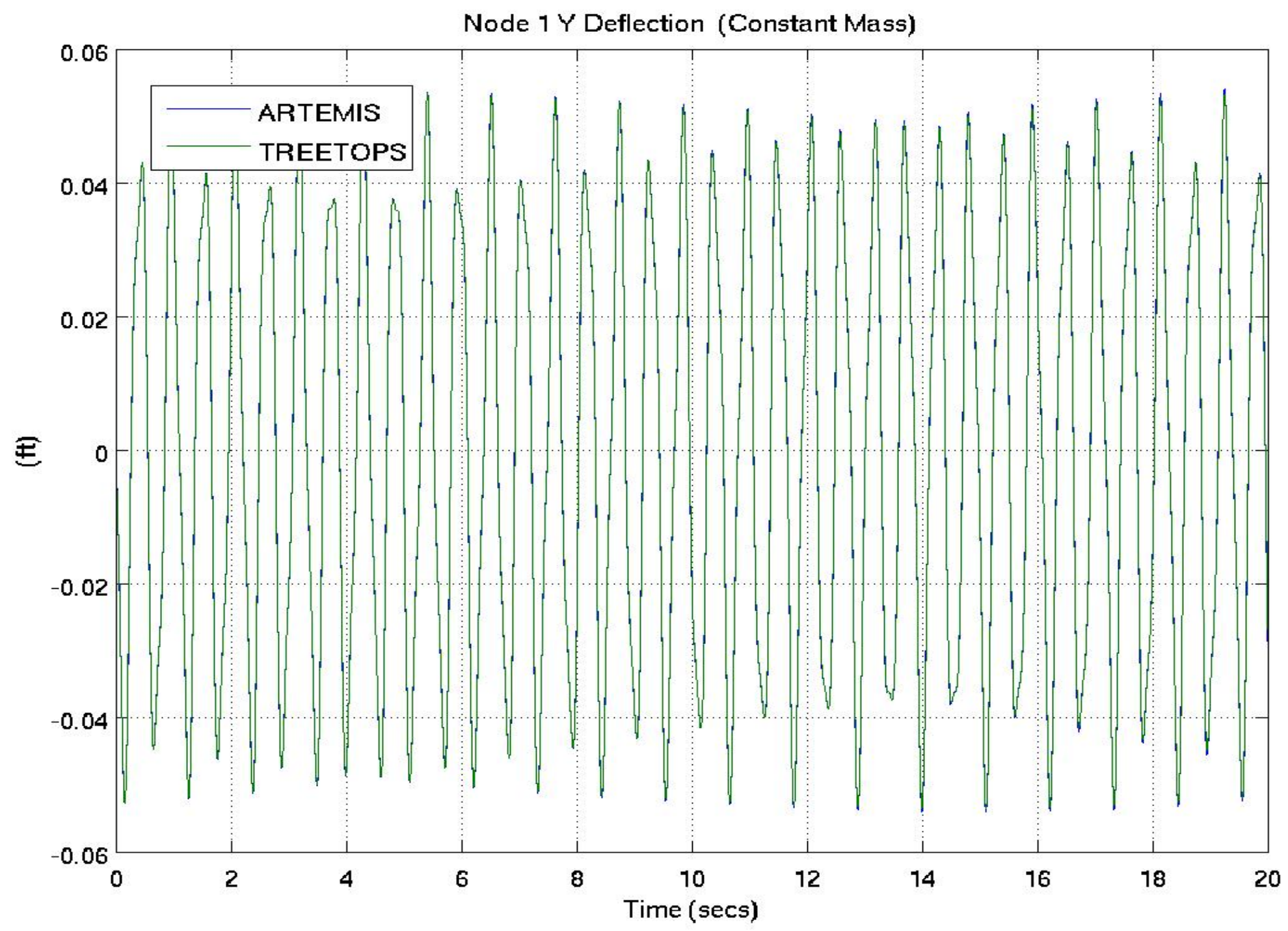




\section{Simulation Results}

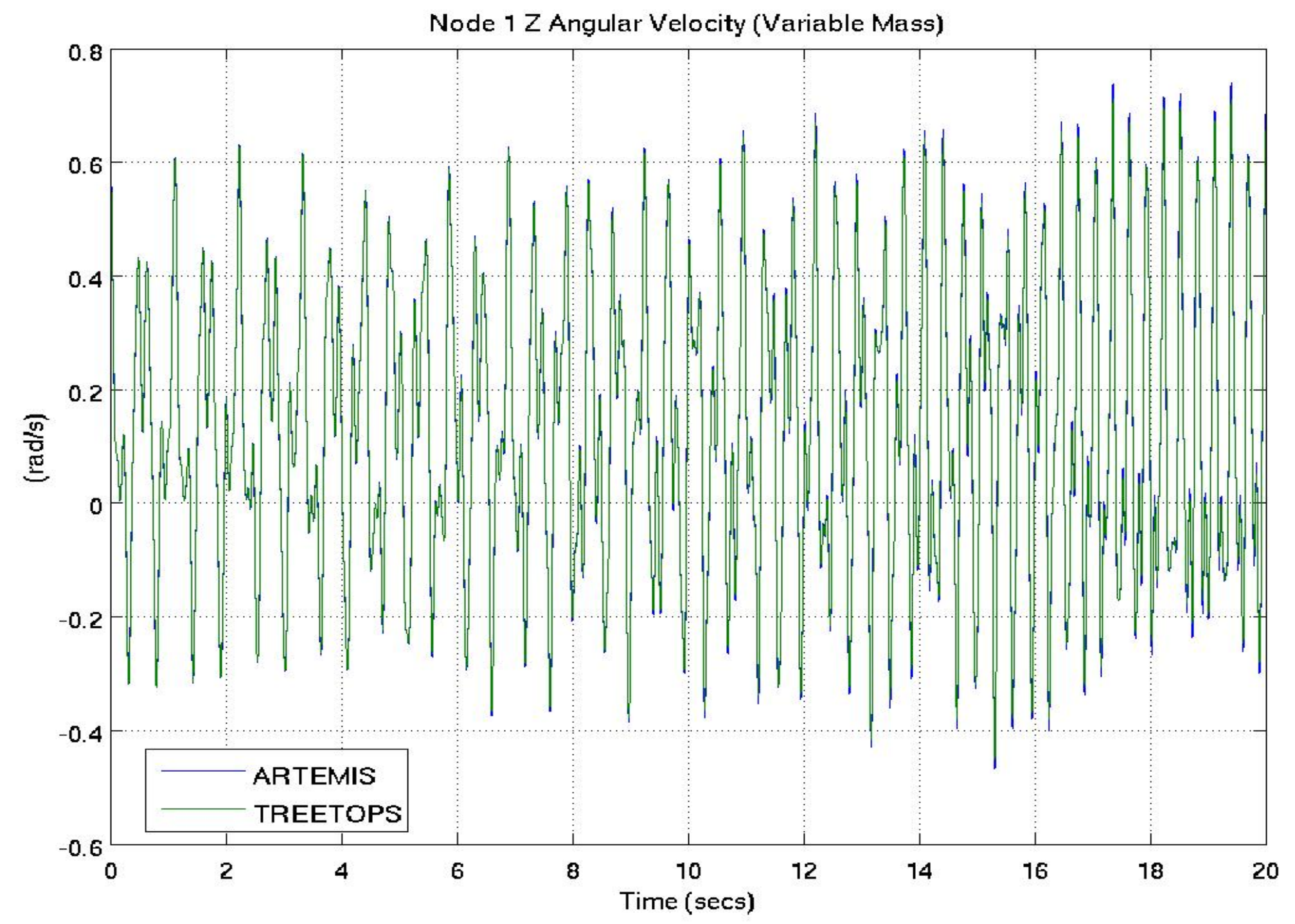




\section{Simulation Results}

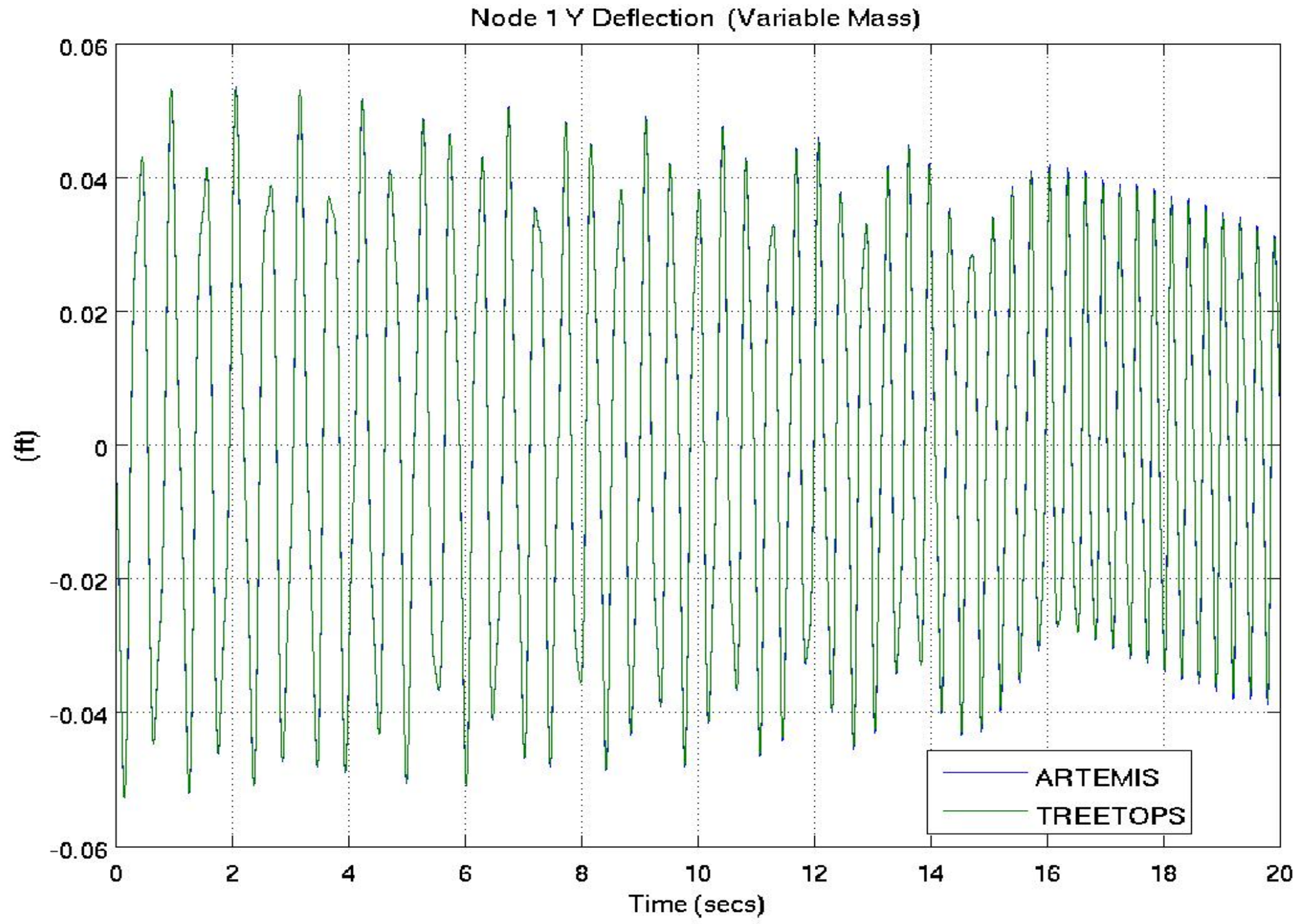


- TREETOPS and ARTEMIS results agree for both constant mass and variable mass configurations using different formulations and models

- System frequencies do not vary for constant mass configuration

- System frequencies vary from 1.8 to $3.3 \mathrm{~Hz}$ for variable mass configuration

- No discontinuities in simulated system response as frequencies change 


\section{Future Work}

- Investigate modal selection techniques for ARTEMIS based on transfer functions

- Evaluate use of constraint and attachment modes to augment free-free vehicle modes

- Clarify "modal damping" for non-orthogonal basis vectors 


\section{References}

- Tobbe, P. A., "An Application of Component Modes to the Flexible Multibody Simulation Problem," Ph.D. Dissertation, Dept. of Mechanical and Aerospace Engineering, Univ. of Alabama in Huntsville, Huntsville, AL, 1995.

- Wilson, H. E., "Dynamics of Variable Mass Flexible Bodies with Time Varying Mode Shapes." M.S. Thesis, Dept. of Mechanical and Aerospace Engineering, Univ. of Alabama in Huntsville, Huntsville, AL, 2007.

- User's Manual for TREETOPS, Rev. 8, Dynacs Inc, Clearwater, FL, 1990.

- Craig, R. R., Structural Dynamics: An Introduction to Computer Methods, John Wiley \& Sons, New York, 1981.

- Goto, K., van de Geijn, R. A., "Anatomy of High-Performance Matrix Multiplication," ACM Transactions on Mathematical Software, Vol. 34, No. 3, 2008, Article 12. 